\title{
Compartmental residence time estimation in batch granulators using a colourimetric image analysis algorithm and Discrete Element Modelling
}

\author{
Andrew D. McGuire ${ }^{1}$, Kok Foong Lee ${ }^{1}$, Maksym Dosta ${ }^{2}$, Sebastian \\ Mosbach $^{1}$, Jan-Georg Rosenboom ${ }^{1}$, Stefan Heinrich ${ }^{2}$, Markus Kraft ${ }^{1,3}$
}

released: 16 June 2016

${ }^{1}$ Department of Chemical Engineering and Biotechnology University of Cambridge New Museums Site

Pembroke Street Cambridge, CB2 3RA

United Kingdom

E-mail: mk306@cam.ac.uk

\author{
${ }^{2}$ Institute of Solids Process Engineering \\ and Particle Technology \\ Hamburg University of Technology \\ Denickestrasse 15 \\ 21073 Hamburg \\ Germany \\ E-mail: stefan.heinrich@tuhh.de
}

3 School of Chemical and Biomedical Engineering

Nanyang Technological University

62 Nanyang Drive

Singapore 637459

E-mail: mk306@cam.ac.uk

Preprint No. 158

\section{UNIVERSITY OF}

CAMBRIDGE 


\section{Edited by}

Computational Modelling Group

Department of Chemical Engineering and Biotechnology

University of Cambridge

New Museums Site

Pembroke Street

Cambridge CB2 3RA

United Kingdom

Fax:

E-Mail:

$+44(0) 1223334796$

c4e@cam.ac.uk

World Wide Web: http://como.cheng.cam.ac.uk/ 


\section{Highlights}

- A colourimetric algorithm to estimate particle concentrations is presented.

- The algorithm is applied to a batch granular mixing system.

- A mixing model is derived to extract local particle residence times.

- Discrete Element Method simulations of the mixing system are carried out.

- Simulation results are compared with those of the experiment.
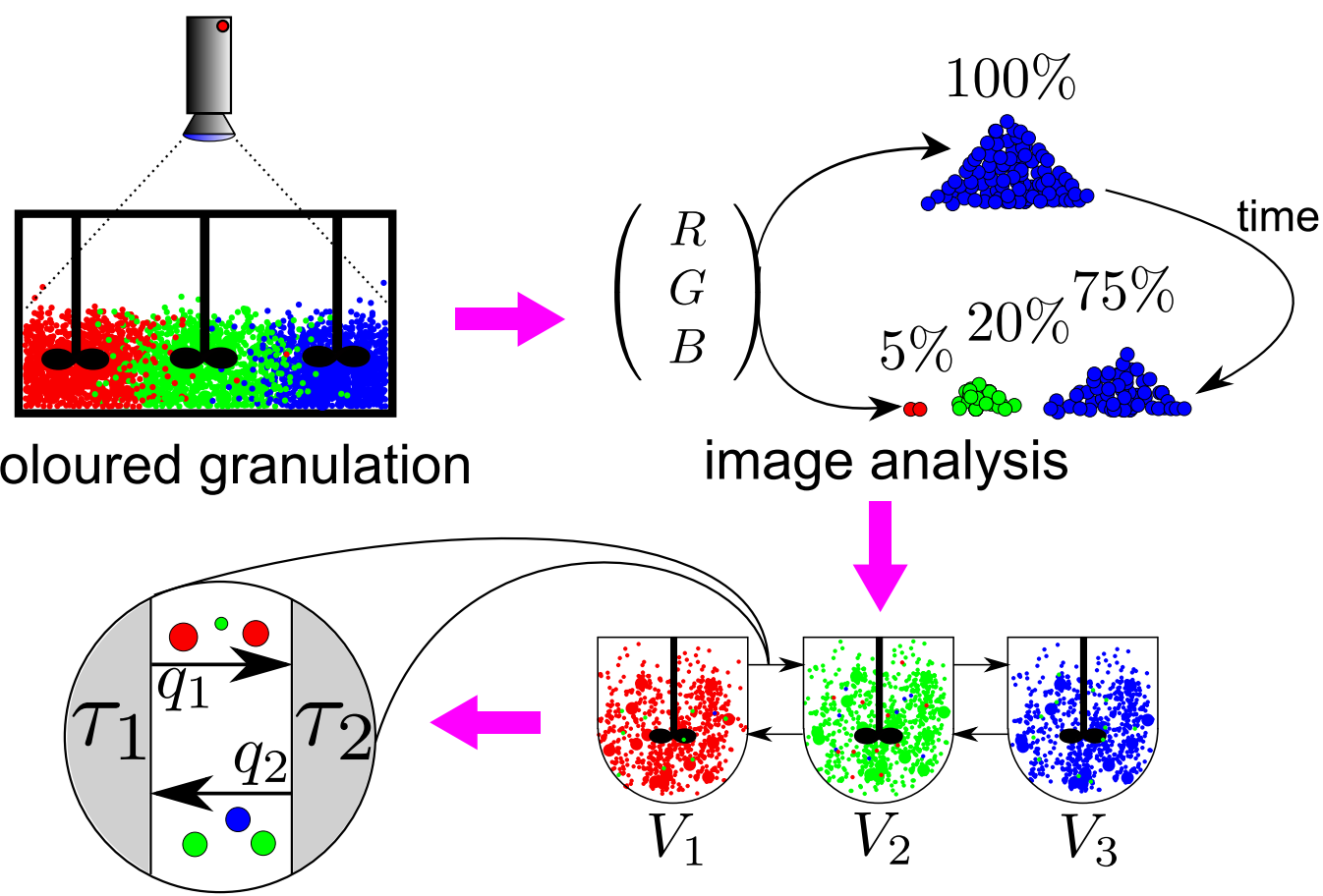

residence time data

mixing model 


\begin{abstract}
In this paper we present an experimental technique and a novel colourimetric image analysis algorithm to economically evaluate particle residence times within regions of batch granulators for use in compartmental population balance models. Residence times are extracted using a simple mixing model in conjunction with colourimetric data. The technique is applied to the mixing of wet coloured granules (binary and tertiary systems) in a laboratory scale mixer. The resulting particle concentration evolutions were in qualitative agreement with those from the mixing model. It was seen that the algorithm was most stable in the case of the binary colour experiments. Lastly, simulations using the Discrete Element Method (DEM) were also performed to further validate the experimental results. Particle concentrations from the simulations were in good agreement with the experimental results.
\end{abstract}




\section{Contents}

1 Introduction 4

2 Methodology 6

2.1 Experimental set-up . . . . . . . . . . . . . . . . . 6

2.2 Image analysis algorithm . . . . . . . . . . . . . . . . 7

2.2.1 Mixer removal algorithm . . . . . . . . . . . . . 8

2.2.2 Construction of the pure particle colour vector set . . . . . . . 8

2.2.3 Decomposition algorithm . . . . . . . . . . . . 10

2.2.4 Model Equations . . . . . . . . . . . . . . . . . . . 11

2.2.5 Fitting and calibration algorithms . . . . . . . . . . 12

2.3 DEM simulation conditions . . . . . . . . . . . . . . . . . . 14

2.3.1 Analysis of DEM results . . . . . . . . . . . . . . 15

$\begin{array}{lll}3 & \text { Results } & 17\end{array}$

3.1 Wet granule experiments . . . . . . . . . . . . . . 17

3.1 .1 Tertiary system . . . . . . . . . . . . . 17

3.1 .2 Binary system $\ldots \ldots \ldots \ldots \ldots \ldots$

3.2 DEM results . . . . . . . . . . . . . . . . . . . . 24

3.2.1 Appropriateness of examining particle concentrations from the

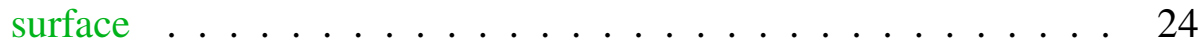

3.2.2 Comparison with experimental data . . . . . . . . . 25

4 Conclusions $\quad 31$

$\begin{array}{ll}\text { References } & \mathbf{3 6}\end{array}$ 


\section{Introduction}

Granulation is an example of particle manufacture and is a key process used in the production of foodstuffs, pharmaceutical tablets and fertilisers among others [35]. The granulated product will have an optimum size (typically a distribution), porosity, solubility, mechanical strength, shape and flow-ability amongst other properties dictated by the specific application. Granules have several advantages over a simple mixture of the raw ingredients such as better flow-ability; better transport properties (such as limited separation of components and reduced risk of powder explosions); dissolution behaviour and controlled release of Active Pharmaceutical Ingredients (API) [10, 61].

Due to the complexity of granulation as a unit operation and the number of particles involved, Population Balance Models (PBM) (Ramkrishna [51]) are typically employed to simulate these systems $[4,9-15,33,48,54-56]$. Here, the individual particle subprocesses such as coagulation, breakage etc. are recast as transformations which act on the particle ensemble to evolve it in time. In this study we define particle as any free moving material in the mixer including unbounded precursor powder and agglomerates.

Vital to such models is the compartmentalisation of the processing system, granulators being just one example, to represent spatial inhomogeneities in processing conditions [5, 29, 34, 43-45, 47]. Each compartment is assumed to be well-mixed and may have a different set of sub-process rates. Material may be transferred between compartments as illustrated in Figure 1. Though large regions of batch granulators cannot generally be considered to be well-mixed at all times during the mixing process, this assumption is nevertheless critical in compartmental population balance models of granular systems. Hence, in this study, we assert the well-mixed assumption on the models used to estimate residence times from the experimental data. Due to this simplification we are primarily interested evaluating the order of magnitude of such residence times in the experimental system.

There is a rich history of mixing analysis in ploughshare mixers. Originally such analysis was performed using positron emission particle tracking (PEPT), in which a radioactive tracer is tracked as the particle bed is mixed. PEPT studies have been used to assess the effect of fill level, particle size and rotor speed in ploughshare mixers and other batch mixing equipment $[18,19,28,37,41,42]$. The estimation of residence times in regions of some system has been attempted using PEPT but the ability to track only a single particle generally leads to large degrees of uncertainty in the results [18, 19]. Generally these studies focus on the quantification of overall mixing within the entire system by means of a mixing index [50]. Whether or not the movement of the radioactive tracer used in PEPT studies is in fact representative of the bulk material is still controversial.

Particle mixing analysis has also been performed using the Discrete Element Methods (DEM)[6, 58]. DEM has allowed for the investigation of variables beyond traditional PEPT, such as the effect of particle shape on mixing [22, 24]. It also allows for the degree of mixing to be assessed qualitatively/quantitatively using many particles, as opposed to the single tracer used under PEPT. Mixing effectiveness in DEM is generally assessed by tagging particles with a colour [7]. Particles are initially segregated by colour and the method of centroids is used to produce mixing curves [22, 23, 42]. Colour particles are 
also used to aid qualitative visual assessment of mixing in such systems [2, 53].

Though both PEPT and DEM allow for in-depth analysis of mixing in batch particulate systems, both have significant drawbacks. Under DEM, it has been stressed that particle transition rates are strongly dependent on the particles physical properties (wet granule strength and asperity sizes), many of which have to be assumed within a DEM simulation $[39,57]$. It has also been noted that mixing characteristics derived from ideal systems (mono-dispersed, spherical, dry materials) have questionable applicability within an industrial context [57]. This highlights the need for mixing experiments to use materials that bear close resemblance (in terms of material physical properties) to those used in application so as to mimic the true granular systems as closely as possible.

Colourimetric methods for the quantitative characterisation of mixing in batch particulate systems have received increased attention in the last decade as imaging equipment such as digital cameras have become relatively inexpensive [1, 3, 52, 57, 62]. Data acquisition through digital imaging is often preferred over more experimentally intensive techniques such as PEPT and computationally intensive techniques such as DEM. In comparison to PEPT experiments, digital camera imaging is widely available, low-cost and does not require irreversible modification of the equipment. Colourimetry has been successfully applied to estimate residence time distributions in continuous granulation systems such as twin screw [40,63] by means of a dye impulse at the binder inlet or a variation in the colour of the feedstock. Here, the residence time distribution is assessed by measuring the time-dependent colour concentrations at the device outlet. Under batch operation the assessment of fluxes/residence time becomes more problematic due to the lack of these inlets/outlets. Santomaso et al. [57] developed a method for quantifying the effective diffusion coefficient (based on a Fickian model) within a batch drum mixer using dry, coloured, non-ideal powders. This employed solidification or freezing of the particle bed prior to extraction and image analysis of axial slices to asses radial concentration gradients. This technique also allowed for a qualitative evaluation of flux patterns within the equipment. The use of coloured tracers has also been extended to the investigation of breakage kinetics in high-shear granulators [49]. Numerous studies have applied colourimetric techniques to assess the global degree of mixing in batch systems $[1,16,30,50,59]$. Again, the mixing is generally quantified by means of a mixing index or centroid evolution. Though such measures are useful in the optimisation of mixing times in powder mixing equipment, they are not easily translated to the corresponding particle fluxes required by compartmental population balance models. To the best of the authors' knowledge, image analysis has not been used to quantify mass flow-rates between different regions of batch mixing equipment, nor has it been applied to wet, non-ideal systems which are subject to particle breakage and coalescence, such as that observed in wet granulation.

In this study we propose a combined experimental/colourimetric image analysis technique for the estimation of volume fraction evolutions within regions of a batch granulator under wet granule conditions. A theoretical framework is then developed to describe the compartmental mixing process and applied to the image analysis data in order to extract compartmental residence times. The same residence time extraction technique is applied to DEM simulations of the mixing system, using various particles sizes distributions and a comparison is made with the experimental approach. 


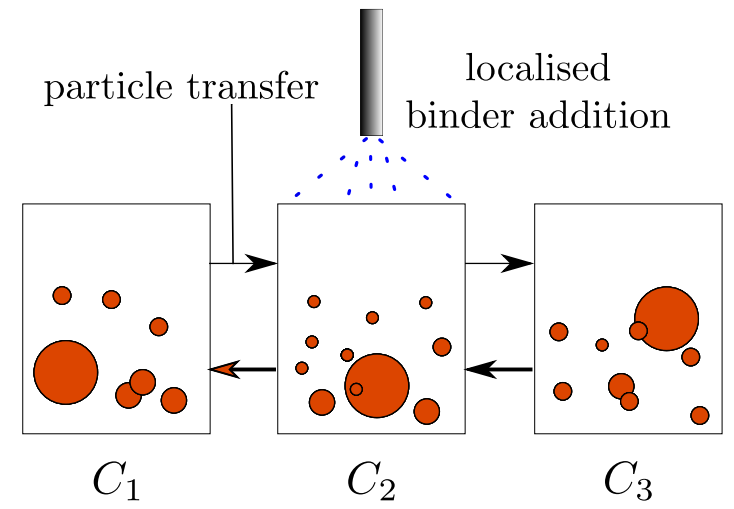

Figure 1: Compartmentalisation of a batch granulation system.

The remainder of the paper is structured as follows: in Section 2 we outline the methodology of the colourimetric mixing experiments and describe, in detail, the image analysis algorithms/mixing models developed to analyse the experimental data. The DEM simulation set-up is then outlined. This is followed by the presentation and discussion of image analysis results for both binary and tertiary coloured, granular systems in Section 3. The DEM results are then presented and compared with those from the experiment. The main findings are then summarised in Section 4.

\section{Methodology}

\subsection{Experimental set-up}

High-shear wet mixing of coloured, granulated Granulac $230 \alpha$-lactose monohydrate (Meggle, Germany) was carried out in a horizontal ploughshare mixer (illustrated in Figure 2). The details and key components of this equipment are described in [38] and [36]. The granulated material used in the mixing experiments was created by granulating $1 \mathrm{~kg}$ of the uncoloured $\alpha$-lactose monohydrate excipient at $120 \mathrm{rpm}$ for 10 minutes in the same mixer. Food colouring was used as the binder fluid. $120 \mathrm{ml}$ of a single food colouring (red, green, blue food colouring, Tesco PLC, UK) was manually added drop-wise to the mixer over the course of the first 2 minutes of the experiment (yielding an approximate liquid-solid mass ratio of 0.12). The granulator was cleaned using warm water and dried between each batch. The mixing experiments were carried out within 6 hours of the formation of the wet granules to ensure the material remained wet. During this time, the granules were spread on drying trays under ambient conditions. The mixing experiments were performed by initially axially partitioning the mixer into three compartments of approximately equal length. $333 \mathrm{~g}$ of each coloured excipient was then decanted into its individual compartment $\left(C_{i}\right)$ as illustrated in Figure 3. The partitions were then removed and the mixer was run at $120 \mathrm{rpm}$ for 10 minutes. Visual recording of the particle bed surface was captured using a digital camera (Canon Powerslide S3) over the course of the experiment. The camera lens was positioned approximately $0.45 \mathrm{~m}$ above the surface 


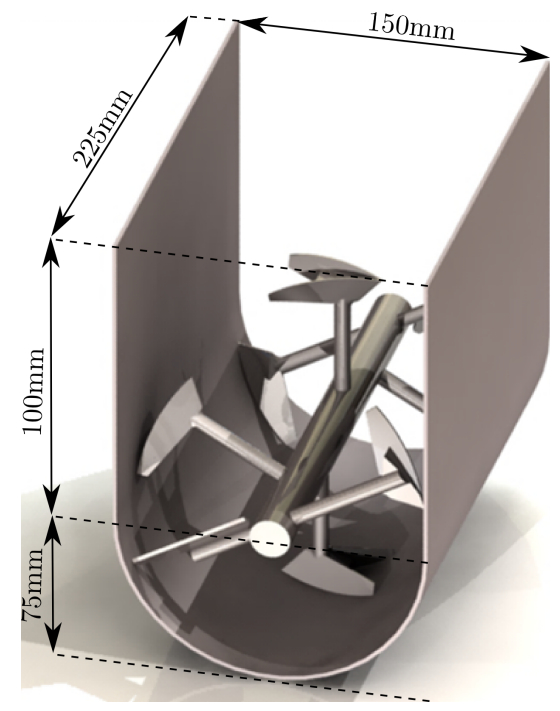

Figure 2: CAD representation of the batch ploughshare mixer used. The ends of the mixer have been removed for clarity.

of the particle bed. The particle bed was uniformly illuminated using a controlled light source (OS12 Fiber Illuminator, Thor Labs). The light source is positioned at the same height as the camera lens and directed towards the bed surface.

Previous work using coloured ballotini and also PEPT experiments with this batch mixer showed that radial dispersion of material is much faster than axial dispersion [17]. This leads to an approximately radially homogeneous system. This fact, coupled with the high packing fraction of the granules (facilitated by the ungranulated powder between granules), allows particle volume fractions to be directly inferred from the particle area fractions observed on the bed surface. The correctness of this assumption has been also proved from obtained DEM simulation results in Section 3.2.

\subsection{Image analysis algorithm}

The video footage acquired from the mixing experiments was split into discrete time image frames and analysed using custom MATLAB [46] routines described in the following section. The image analysis was specifically carried out on the region of the particle bed highlighted in Figure 3. An algorithm was developed to remove the mixer from the analysis were it could be clearly distinguished from the particles within the bed. Additional routines allowed for the translation of RGB values from the image series to particle volume fractions. Finally, fitting of derived volume fraction models was performed to extract characteristic time data from the volume fraction evolutions. A simplified flowchart of the algorithm is illustrated in Figure 4. 


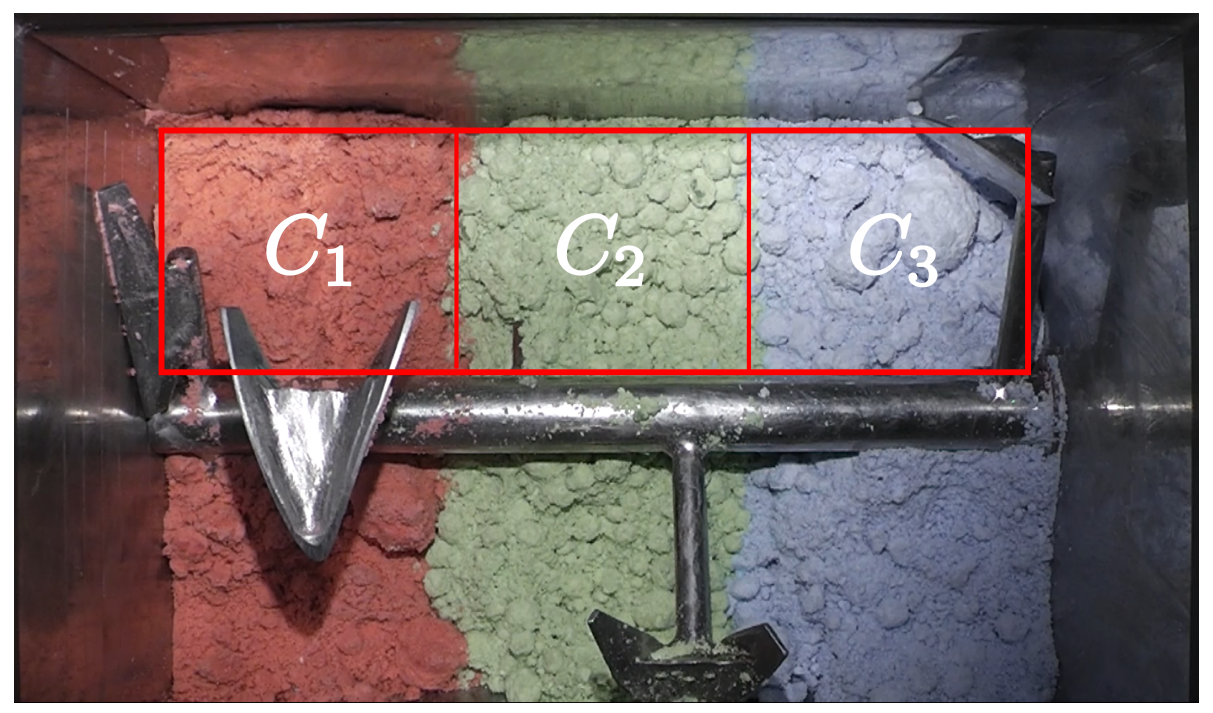

Figure 3: Initially segregated wet granule system with highlighted analysis region. $C_{1}$ : Red particles, $C_{2}$ : Green particles, $C_{3}$ : Blue particles.

\subsubsection{Mixer removal algorithm}

The mixer pixels are removed firstly by manually defining two points in RGB space on the first, un-mixed frame of the image series corresponding to a mixer pixel which is highly reflective and another which is shaded. Each of these points is then given a corresponding RGB tolerance resulting in two spheres in RGB space which corresponds to regions of the space typically occupied by pixels containing the mixer blades/boundary. These points and tolerances are manually tuned to remove as many of the mixer pixels from the image series as possible without removing the mixed and unmixed particle pixels. Each pixel in each image frame in the series is then parsed. If the RGB value of a pixel lies within either of the mixer spheres then the RGB value of that pixel is set to unity. These values are then detected and ignored in any further analysis.

\subsubsection{Construction of the pure particle colour vector set}

In the interest of clarity, the measured digital RGB component related variables will from this point onward be denoted by $R, G$ and $B$. Variables related to particle colours will be denoted in subscripts by name as red, green or blue. For each compartment, within each frame in the image time series, the measured RGB vector is averaged over all non-mixer pixels $p$. This average is then normalised to unit vector form to reduce the noise associated with shadows cast by larger particles and the mixer boundaries. The reduced form of the measured RGB vector is defined as an rgb vector. This yields a time dependent vector in 


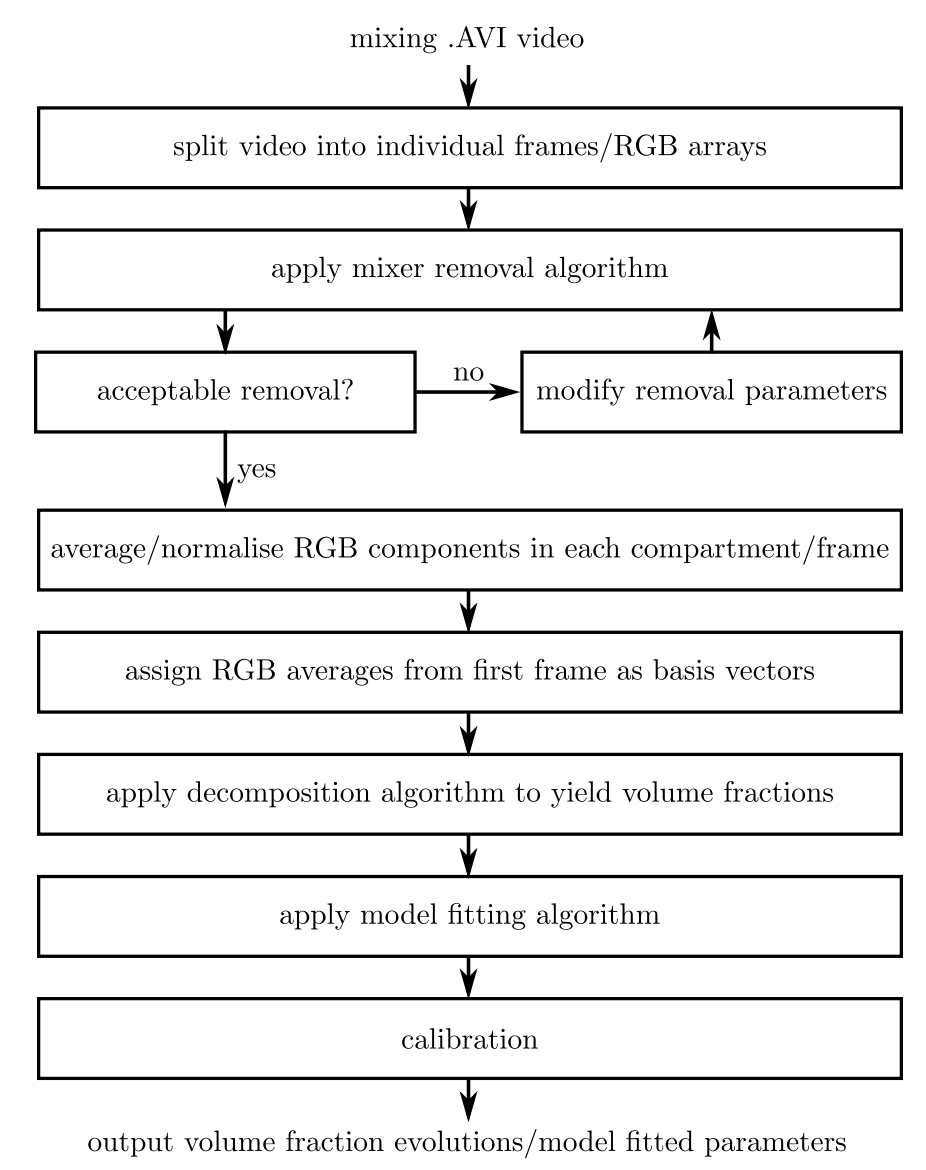

Figure 4: A simplification of the custom image analysis algorithm developed to produce volume fraction evolutions and mixing time characteristics from experimental image recordings.

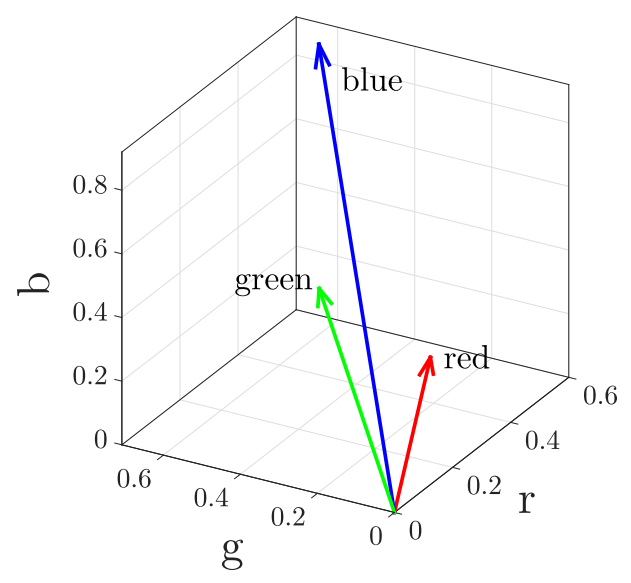

Figure 5: Pure particle colour vectors $\boldsymbol{\lambda}_{i}(0)$ in rgb space corresponding to the three particle colours in the tertiary component wet granule system. 
$\operatorname{rgb}$ space $\boldsymbol{\lambda}_{i}(t)$ for each compartment $i$ given as:

$$
\lambda_{i}(t)=\left(\begin{array}{c}
r_{i}(t) \\
g_{i}(t) \\
b_{i}(t)
\end{array}\right)=\left[\left(\sum_{p} R_{p}(t)\right)^{2}+\left(\sum_{p} G_{p}(t)\right)^{2}+\left(\sum_{p} B_{p}(t)\right)^{2}\right]^{-1} \sum_{p}\left(\begin{array}{c}
R_{p}(t) \\
G_{p}(t) \\
B_{p}(t)
\end{array}\right)
$$

At $t=0$ (when the system is unmixed) in an $n$-component, $n$-compartment system the above equation yields $n$ unique pure particle rgb vectors. Given a three component system in which the particle colour vectors are linearly independent, these measured rgb vectors form a basis set for rgb space. The maximum number of components that can be modelled is limited to three (the length of the rgb vector) but the possible number of compartments is, in theory, infinite. An example of the pure particle colour vector set constructed from the wet granule experimental data is shown in Figure 5.

\subsubsection{Decomposition algorithm}

We propose that the observed rgb colour changes $\Delta \boldsymbol{\lambda}_{i}(t)=\boldsymbol{\lambda}_{i}(t)-\boldsymbol{\lambda}_{i}(0)$ within compartment $i$ of an $n$ compartment, $m$ component system can be modelled as a linear summation of all of the pure particle colour vectors $\lambda_{h}(0) h=\{1,2, \ldots, m\}$, each weighted by the corresponding change in volume fraction of the coloured particle that the pure colour vector represents $\Delta v_{i, j}(t)=v_{i, j}(t)-v_{i, j}(0), j=\{\operatorname{red}, \ldots$, colour $\mathrm{m}\}$.

Though it is generally not possible to superimpose colours in RGB colour space [32], preliminary testing showed that colour decomposition could be achieved using the proposed algorithm within the range of colours tested. Generally, known quantities of powders are mixed together to form a calibration curve (typically in grey-scale) which is then used to decompose concentration in subsequent image frames [60]. However, for a tertiary coloured system, a large number of mixing experiments would be required to effectively cover the three-dimensional concentration space. Furthermore, since RGB values are device dependent and sensitive to lighting conditions, the applicability of the calibration curves to further experiments is questionable [60]. For these reasons a pre-calibration approach was not used in this study. In preliminary work, the image data was mapped to alternate colour spaces such as HSL, CIE L*a*b*, CIE XYZ, Y'CbCr and RYB [27, 31]. It was seen that the linear colour decomposition algorithm performed best in the original RGB space and is therefore use for the rest of the study.

Using the proposed decomposition algorithm, given a tertiary particle colour, three compartment system (originally set-up as in Figure 3 ) in some mixed state, then the rgb change in compartment $i\left(\Delta \boldsymbol{\lambda}_{i}(t)\right)$ may be calculated directly from the camera RGB data using Eq. (1). The unknown particle volume fractions in compartment $i\left(v_{i, \text { red }}(t), v_{i, \text { green }}(t)\right.$ and $\left.v_{i, \text { blue }}(t)\right)$ may then be simply calculated by solving:

$$
\left[\begin{array}{lll}
\boldsymbol{\lambda}_{1}(0) & \boldsymbol{\lambda}_{2}(0) & \boldsymbol{\lambda}_{3}(0)
\end{array}\right] \Delta \boldsymbol{\nu}_{i}(t)=\Delta \boldsymbol{\lambda}_{i}(t)
$$

where:

$$
\Delta \nu_{i}(t)=\left[\begin{array}{c}
\Delta v_{i, \text { red }}(t) \\
\Delta v_{i, \text { green }}(t) \\
\Delta v_{i, \text { blue }}(t)
\end{array}\right]
$$


Given $\Delta \boldsymbol{\lambda}_{i}(t)$ from Eq. (1), Eq. (2) is solved for each compartment/frame to yield a discrete volume fraction change time-series $\Delta \nu_{i}(t)$ for each compartment in the system. In the interest of lowering the memory/computational intensity of the analysis, not all frames of the experimental video footage were analysed (1-2 seconds worth of footage were skipped between analysis frames depending on the length and resolution of footage to be analysed).

Preliminary work using the above algorithm showed that, in general, the steady state volume fractions generated by solving Eq. (2) were not as expected. For example, given a 3 compartment, tertiary system with an equal mass of each particle colour, the steady state should exhibit $v_{i, \text { red }}=v_{i \text {,green }}=v_{i \text {,blue }}=1 / 3$ for all compartments $i$. Originally the breakage of this equality in the experimental results was assumed to be a result of the dominance of one of the particle colours over the others by selective coating of larger particles in the dominant colour (layering) to yield false volume fractions. However, preliminary experiments with dry, coloured sand (which does not form agglomerates or exhibit layering) as the mixing medium also exhibited steady state offsets. It could be concluded from this that certain particle colours have a greater effect on the rgb value of the mixture than others. Thus, to obtain 'true' volume fractions at a given time within a compartment, a degree of calibration is required to map the volume fractions found in the solution to Eq. (2) to the true particle volume fractions. From this point on-wards $v_{i, j}$ will be defined as the uncalibrated particle volume fraction and $\hat{v}_{i, \mathrm{j}}$ as the calibrated (true) particle volume fraction of particle type $j$ in compartment $i$. The linear calibration mapping used in this study is:

$$
\Delta \hat{\boldsymbol{\nu}}_{i, \text { red }}(t)=\boldsymbol{\Phi}_{i} \Delta \boldsymbol{\nu}_{i, \text { red }}(t),
$$

where

$$
\Delta \hat{\boldsymbol{\nu}}_{i}(t)=\left[\begin{array}{c}
\Delta \hat{\boldsymbol{v}}_{i, \text { red }}(t) \\
\Delta \hat{v}_{i, \text { green }}(t) \\
\Delta \hat{\boldsymbol{v}}_{i, \text { blue }}(t)
\end{array}\right],
$$

and $\Phi_{i}$ is the calibration array for compartment $i$, defined as:

$$
\boldsymbol{\Phi}_{i}=\left[\begin{array}{ccc}
\phi_{i, \text { red }} & 0 & 0 \\
0 & \phi_{i, \text { green }} & 0 \\
0 & 0 & \phi_{i, \text { blue }}
\end{array}\right]
$$

The evaluation of the calibration constants $\phi$ is discussed later in this section.

\subsubsection{Model Equations}

A model for each particle volume fraction evolution in each compartment is derived by considering an idealised reactor network. A complete derivation of the model and its solution is presented in Appendix I. In the model, each compartment is assumed to be perfectly mixed, with a tertiary particle colour system initially loaded as [red, green, blue]. It assumed that the separation between compartment boundaries is sufficiently large and the time step between analysis frames is sufficiently small such that particles 
cannot 'skip' over compartments (e.g. from $C_{1}$ directly to $C_{3}$ ). The model also assumes constant mass, constant particle density and constant particles size in each compartment hold-up throughout the mixing process. The model was solved for the particle volume fraction change evolutions $\Delta \hat{v}_{i, \mathrm{j}}(t)$ for each colour $j$ in each compartment $i$ to yield four unique model equations:

$$
\begin{aligned}
\Delta \hat{v}_{1, \text { red }}(t)_{\text {model }} & =\left[\frac{1}{2} \exp \left(-\frac{(t-\theta)}{\tau_{1}}\right)+\frac{1}{6} \exp \left(-\frac{3(t-\theta)}{\tau_{1}}\right)-\frac{2}{3}\right] H(t-\theta), \\
\Delta \hat{v}_{2, \text { red }}(t)_{\text {model }} & =\left[-\frac{1}{3} \exp \left(-\frac{3(t-\theta)}{\tau_{1}}\right)+\frac{1}{3}\right] H(t-\theta), \\
\Delta \hat{v}_{3, \text { red }}(t)_{\text {model }} & =\left[-\frac{1}{2} \exp \left(-\frac{(t-\theta)}{\tau_{1}}\right)+\frac{1}{6} \exp \left(-\frac{3(t-\theta)}{\tau_{1}}\right)+\frac{1}{3}\right] H(t-\theta), \\
\Delta \hat{v}_{2, \text { green }}(t)_{\text {model }} & =\left[\frac{2}{3} \exp \left(-\frac{3(t-\theta)}{\tau_{1}}\right)-\frac{2}{3}\right] H(t-\theta),
\end{aligned}
$$

where $\tau_{1}$ is the residence time of compartment $1, H$ is the Heaviside step function. $\theta$ is the pre-defined time delay used to model the system prior to turning on the mixer such that the stability of the image analysis algorithm can be further assessed. Due to the symmetrical nature of both the mixture operation and loading it is known that in an ideal system:

$$
\begin{aligned}
\hat{v}_{1, \text { blue }}(t) & =\hat{v}_{3, \text { red }}(t), \\
\hat{v}_{2, \text { blue }}(t) & =\hat{v}_{2, \text { red }}(t), \\
\hat{v}_{3, \text { blue }}(t) & =\hat{v}_{1, \text { red }}(t), \\
\hat{v}_{1, \text { green }}(t)=\hat{v}_{3, \text { green }}(t) & =\hat{v}_{2, \text { red }}(t)=\hat{v}_{2, \text { blue }}(t),
\end{aligned}
$$

and

$$
\tau_{1}=2 \tau_{2}=\tau_{3}
$$

We now have a complete set of equations to describe the calibrated volume fraction evolution of each particle colour in each compartment of the system. These are graphically illustrated for selected parameter values in Figure 20. These models are easily combined to model binary particle colour systems and a variety of loading patterns. Furthermore, such a modelling procedure could also be carried out on alternative/more complex granulation/mixing equipment.

\subsubsection{Fitting and calibration algorithms}

As previously mentioned, it is known that the colour change associated with a certain change in particle volume fraction requires a degree of calibration in order to achieve the expected steady state results. Since the measured steady state volume fraction of a given particle type in a certain compartment is not known a priori, an additional fitted parameter $K_{i, j}$ is introduced to the model equations (the reader is reminded that $i$ refers to the compartment index and $j$ to the particle colour). The $K_{i, j}$ parameter controls the total volume fraction change at steady state $\left(\Delta v_{i, j}(\infty)_{\text {model }}\right)$ when the volume fraction dynamics have died out. We defined the uncalibrated particle volume fraction models as

$$
\Delta v_{i, j}(t)_{\text {model }}=K_{i, j} \Delta \hat{v}_{i, j}(t)_{\text {model }} .
$$




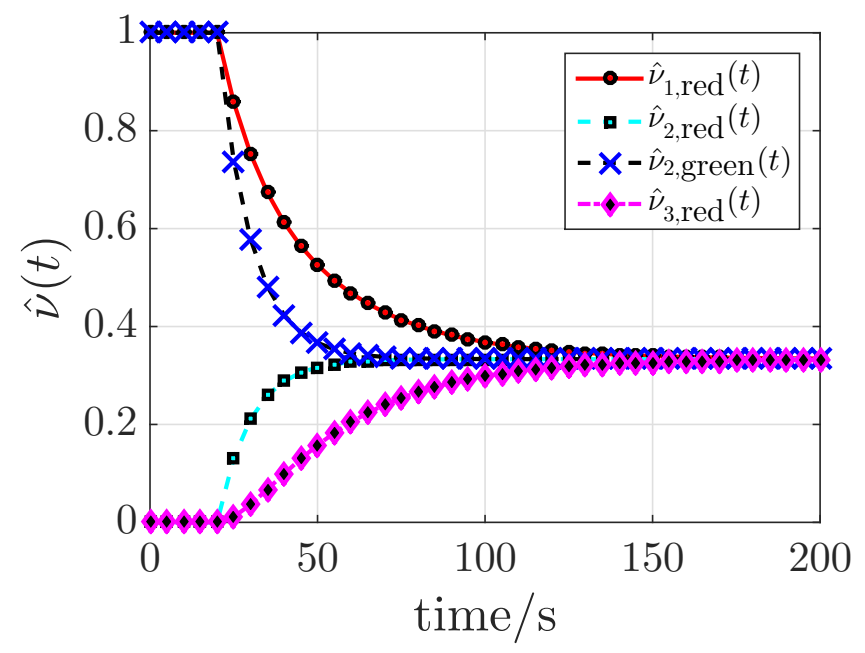

Figure 6: Theoretical model responses from Eqns. (7)-(10) for a tertiary system initial loaded from left to right as [red, green,blue] with $\tau_{1}=30 \mathrm{~s}$ and $\theta=20 \mathrm{~s}$.

It is important to note that the compartment residence times $\tau_{1}$ can be directly extracted from the fitted, uncalibrated volume fraction models. Eq. (13) need only be solved for $\hat{v}_{i, j}(t)_{\text {model }}$ if we wish to know the true volume fraction of a given particle type at a particular time within a compartment.

The mapping of the uncalibrated measured volume fractions $v_{i, j}(t)$ to the calibrated measured volume fractions $\hat{v}_{i, j}(t)$ also requires knowledge of the steady state change in the uncalibrated volume fraction. In theory this could be done for each volume fraction evolution by observing the final volume fraction value $v_{i, j}\left(t_{\text {end }}\right)$ from the discrete, measured volume fraction times-series. However, since the measured data is subject to experimental noise it is more suitable to estimate the measured steady state volume fraction $v_{i, j}(\infty)$ from the fitted model response $v_{i, j}(t)_{\text {model }}$. Ultimately we are interested in evaluating the calibration coefficients for the measured volume fraction data introduced in Eq. (6). From Eq. (4)-(6) we see:

$$
\hat{v}_{i, j}(t)=\phi_{i, j} v_{i, j}(t)
$$

As mentioned above, to avoid the noise associated with the experimental data we assume a good model fit such that:

$$
v_{i, j}(t)=v_{i, j}(t)_{\text {model }} .
$$

It is also desired that our calibrated model and calibrated measured volume fraction data are in agreement i.e.:

$$
\hat{v}_{i, j}(t)_{\text {model }}=\hat{v}_{i, j}(t) .
$$

Together, Eq. (13)-(16) show that the calibration constant for a particle of type $j$ in compartment $i$ may be simply related to the fitted gain of the corresponding fitted volume fraction model $K_{i, j}$ as:

$$
\phi_{i, j}=K_{i, j}^{-1}
$$


Again the calculated residence times $\tau$ are unaffected by the calibration process and such calibration is only required if evaluation of the 'true' volume fractions is desired.

The steps of fitting/calibration procedure is summarised as follows:

1. The discrete rgb time series $\Delta \boldsymbol{\lambda}_{i}(t)$ is generated from the measured RGB camera data using Eq. (1) for all compartments $i$.

2. Eq. (2) is then solved for all $0 \leq t \leq t_{\text {end }}$ to yield the discrete, uncalibrated, measured volume fraction time series $v_{i, j}(t)$ for all particle types $j$.

3. For each $j$ the appropriate model $\Delta \hat{v}_{i, j}(t)_{\text {model }}$ is then selected from Eqs. (7)-(10).

4. The model equations to be fitted are then given by (13).

5. By manually estimating the point at which mixer is turned on within the experimental video footage and starting the image analysis $20 \mathrm{~s}$ prior to this event, $\theta$ is set to approximately $20 \mathrm{~s}$ (or the closest time for which an image frame exists).

6. For each $j, \Delta v_{i, j}(t)_{\text {model }}$ is then fitted to the associated discrete time-series $\Delta v_{i, j}(t)$ using the MATLAB fittype function [46] - the fitting parameters being $\tau_{1}$ and $K_{i, j}$. At this point we have the fitted residence time of compartment 1 (from which all others may be calculated by way of Eq. (12)) based on all $j$ components in compartment $i$.

7. The calibrated ('true'), measured volume fractions are evaluated using Eq. (4),(17), the discrete, uncalibrated, measured volume fraction time series $v_{i, j}(t)$ and the fitted model parameters $K_{i, j}$.

8. The calibrated volume fraction model responses are given by Eq. (13).

9. Steps 2-7 are repeated for each compartment $i$.

\subsection{DEM simulation conditions}

The DEM implementation in this paper uses the Hertz-Mindlin model for the calculations of both the particle-particle and particle-wall contact. For more details regarding the implementation, readers can refer to [26]. The geometry used in the DEM simulations were constructed to match the laboratory mixer and the CAD drawing is shown in Figure 2.

Dry spherical particles are used in the simulations and the effects of binder liquid are ignored. As past studies have shown that particle size distribution (PSD) has a significant influence over the flow behaviour [20,21], two simulations with different PSDs are performed for comparison with the image analysis experiments. The first simulation consists of uniformly sized particles with the diameter of $2.6 \mathrm{~mm}$, whereas the particles in the second simulation are generated according to the size distribution of particles used in the image analysis experiments. The PSD is measured by drying and sieving the particles, more details regarding the experimental procedure can be obtained in [38]. In the measured distribution, the particle size ranges from $53 \mu \mathrm{m}$ to $2 \mathrm{~cm}$. In order to save the computational cost of the simulation, a lower cut off size of $1.7 \mathrm{~mm}$ is used to reduce 
the number of particles to simulate. The numbers of particles in both DEM simulations are adjusted to match the fill level used in the experiments which resulted in about 80000 particles for both cases.

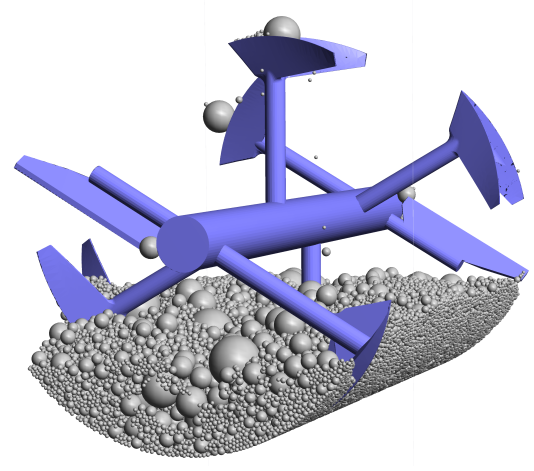

(a) DEM with PSD.

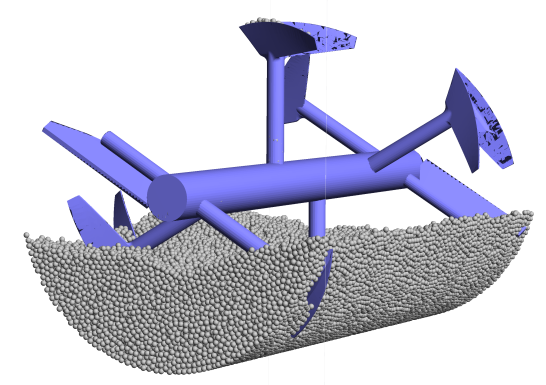

(b) DEM with uniform particles.

Figure 7: Initial states of the DEM simulations.

The material properties used for the simulations are summarised in Table 1. Among the properties, the density, coefficient of restitution and Young's Modulus are experimentally measured, and the rest are extracted from the literature. Dried lactose granules are used for the measurements and the effects of binder are ignored. The coefficient of restitution of dried lactose granules is measured using an optical technique described in [25] and an average value of 0.2 is obtained from the measurements. On the other hand, the Young's Modulus is calculated from single granule compression tests described in [54]. An average value of $1.7 \times 10^{7} \mathrm{~Pa}$ is used for the simulation with uniform particle size and the values used in the simulation with PSD are shown in Figure 8 where the Young's Modulus is set according to the particle size.

Table 1: Material properties used in DEM. Values shown are for both simulations with uniform particle and PSD unless otherwise stated.

\begin{tabular}{lc}
\hline Property & Value \\
\hline Density of lactose & $1545 \mathrm{~kg} \mathrm{~m}^{-3}$ \\
Coefficient of restitution & 0.2 \\
Coefficient of friction (particle-particle) [8] & 0.2 \\
Coefficient of friction (particle-wall) [8] & 0.35 \\
Poisson ratio [8] & 0.3 \\
Young's Modulus of lactose (uniform size) & $1.7 \times 10^{7} \mathrm{~Pa}$ \\
Young's Modulus of lactose (PSD) & Refer Figure 8 \\
Young's Modulus of steel [8] & $2.15 \times 10^{11} \mathrm{~Pa}$ \\
\hline
\end{tabular}

\subsubsection{Analysis of DEM results}

Since the positions of each particle are available from the DEM simulations, it is possible to calculate the exact colour volume fractions for each compartment. At time $0 \mathrm{~s}$, the 


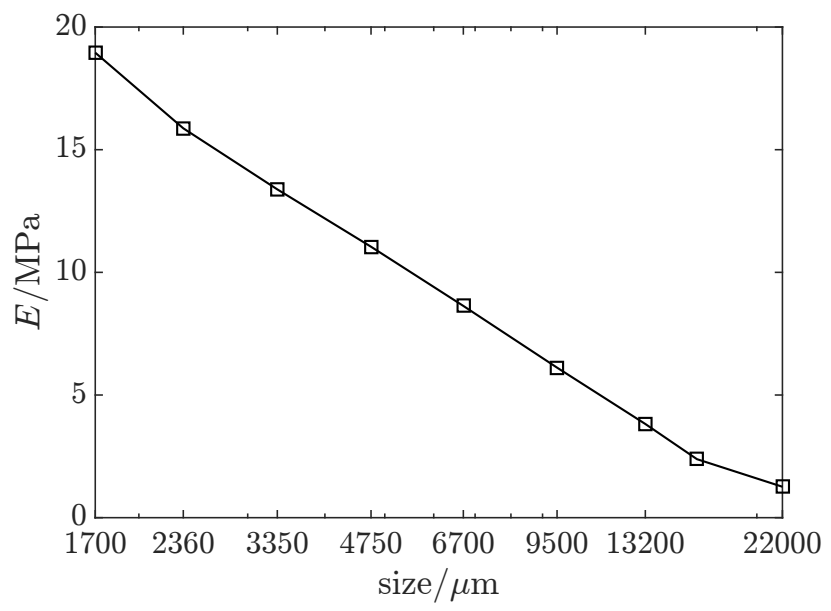

Figure 8: Young's Modulus, E, used in the DEM simulation with PSD.

particles in each compartment are tagged with colours $\left(C_{1}\right.$ : red, $C_{2}$ : green, $C_{3}$ : blue $)$ as shown in Figure 14. Then, the volume fraction of the colour $j$ in compartment $i$ at time $t$ is calculated by

$$
\hat{v}_{i, j}(t)_{\mathrm{DEM}}=\frac{V_{\text {particle }}(t, i, j)}{\sum_{j} V_{\text {particle }}(t, i, j)},
$$

where $V_{\text {particle }}(t, i, j)$ is the total volume of particles of the colour $j$ in compartment $i$ at time $t$ and $\sum_{j} V_{\text {particle }}(t, i, j)$ is the total volume of all the particles which is located in compartment $i$ at time $t$. 


\section{Results}

\subsection{Wet granule experiments}

\subsubsection{Tertiary system}

The resulting measured volume fraction evolutions (both calibrated and uncalibrated) derived from the image analysis algorithm applied to the tertiary, 3 compartment, wetgranule system (initially loaded from $C_{1}$ to $C_{3}$ as [red, green, blue]) are shown in Figure 10 (including the corresponding fitted model responses). The fitted model parameter values are presented in Figure 9. It is noted from Figure 10 (b) and (d) that the calibration procedure has failed due the high noise to $\Delta v(\infty)$ ratio in the uncalibrated blue volume fraction evolutions (Figure 10 (a) and (c)), resulting in a poor model fit. The noise in the uncalibrated blue particle volume fraction data has been amplified as the calibration procedure has heavily weighted small changes in $v_{i \text {,blue }}$ in order to drive $\hat{v}_{i \text {,blue }}(\infty)$ to the expected value of $1 / 3$. This undesired amplification could potentially be reduced by time-averaging the measured volume fraction data. A slight degree of colour identification error between the blue and red particles is observed in uncalibrated central compartment (Figure 10(c)), reflected in the large disparity in $\tau_{1}$ values between red and green components in $C_{2}$. This could be the results of a linear dependency between the pure particle vectors $\lambda_{1}(0)$ and $\lambda_{3}(0)$ since the pure components occupy a relatively small region of RGB space. It is noted that a perfect model fit to the measured data was assumed in the derivation of the calibration constants used to translate between $v_{i, j}(t)$ and $\hat{v}_{i, j}(t)$. Hence, the poor fit achieved in Figure 10 (a) and (c) has resulted in the calibrated, measured blue particle volume fractions not achieving the desired steady state value in Figure 10 (b) and (d).

From Figure 9 it is noted that a high degree of calibration $\left(K_{i, j}=1\right.$ implies no calibration was required) is required in all cases. It is noted from Figure 10(f) that low noise to $\Delta v(\infty)$ ratio has allowed for much better calibration of the volume fractions and that this plot is in very good qualitative agreement with the trends predicted by the model evolutions in Figure 20. However, it is observed that there is a disparity between the fitted $\tau$ values in $C_{3}$ in terms of particle colour (or alternatively, initial position). There are several reasons why this may be the case: firstly, it was theorised that the relationship between $\Delta \hat{v}_{i, j}$ and $\Delta \lambda_{i}$ is not purely linear as assumed in Eq. 2. As such, though the initial and final conditions of the calibrated volume fractions reflect the continuity of the system (the latter being a direct result of the calibration procedure) a degree of apparent non-linearity/material discontinuity may exist between the two steady states. However, a plot of the total volume fraction within each compartment as a function of time (Figure 11) shows very limited deviation in terms of continuity throughout the experiment in the case of $C_{3}$. The large fluctuations in total volume fraction within $C_{1}$ and $C_{3}$ are the result of the failure in the calibration of the blue particle volume fractions within these compartments. This indicates limited non-linearity in the RGB mixing (in cases where limited colourimetric noise/colour identification errors exist) and supports the linear colourimetric relation (Eq. 2) and calibration procedure proposed.

A second factor which has the potential to generate the disparity between $\tau$ values in $C_{3}$ 
is the differential layering rate of each particle type. Since each colour of food colouring consists of a slightly different formulation, the physical properties of the resulting granular material can vary. If the resulting granular product of one colour was more prone to breakage than the others then the selective binding of subsequent 'loose' material on to large particles of alternate colours would lead to an over estimation of the volume fraction of the particle type that was prone to breakage. Though the layering of loose material on to the surface of larger particles was observed during the experiment, the existence of selective layering could not be conclusively confirmed from these observations. This factor is not incorporated into the calibration procedure.

However, the factor likely to have the most significant influence on the disparity between $\tau$ values in $C_{3}$ is the breach of the well mixed compartment assumption used in the model construction. Since the compartments in the experimental system are generally not wellmixed, particularly in the initial stages, it is noted that, for the volume fraction of the blue particle to decay in $C_{3}$ it needs only to exchange material across the boundary between $C_{2}$ and $C_{3}$. Thus green material needs only to cross the boundary, and not necessarily become well-mixed within $C_{3}$ to reduce $\hat{v}_{3, \text { blue }}$ therein. On the other hand, for red material to travel between $C_{1}$ and $C_{3}$ it must first disperse across $C_{2}$. $C_{2}$ becomes well mixed in terms of the red component during this process, hence the $\tau_{1}$ value derived from the red component in $C_{3}$ is greater than both the blue and green particle values. The validity of the 'well-mixed' compartment assumption could be improved by reducing the compartment size/increasing the number of compartments. Nevertheless, this raises the question of which $\tau$ value is most appropriate for use in a compartmental simulation of the system. Since the existing compartmental population balance models for such system generally assume compartments to be well-mixed it would seem reasonable to derive $\tau$ values from the red particle evolution in $C_{3}$ since this incorporates a transition through a well-mixed compartment. This gives $\tau_{1} \approx 41 \mathrm{~s}$. 


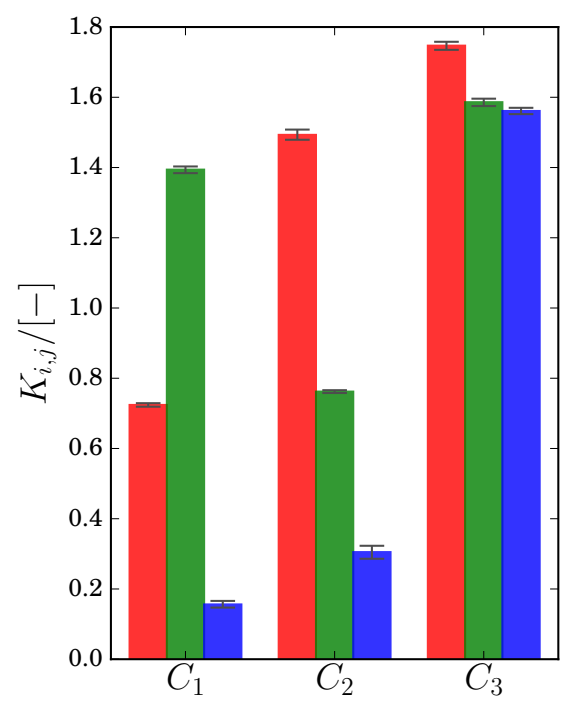

(a)

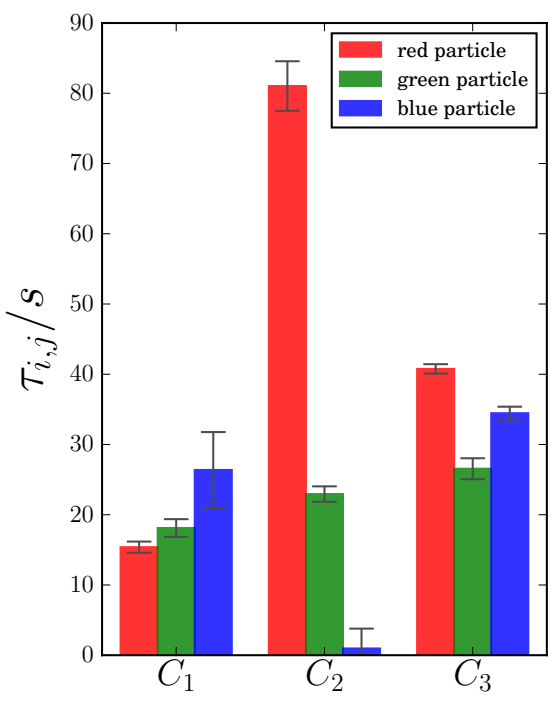

(b)

Figure 9: Fitted model parameters derived from the tertiary component mixing experiment with 95\% confidence intervals. 


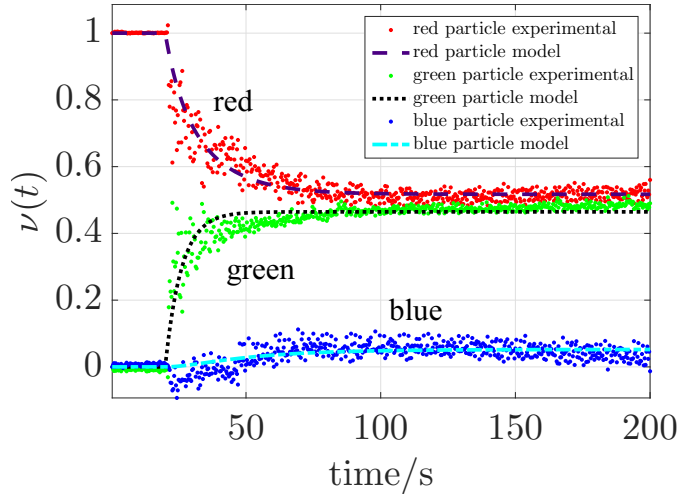

(a) $C_{1}$ uncalibrated.

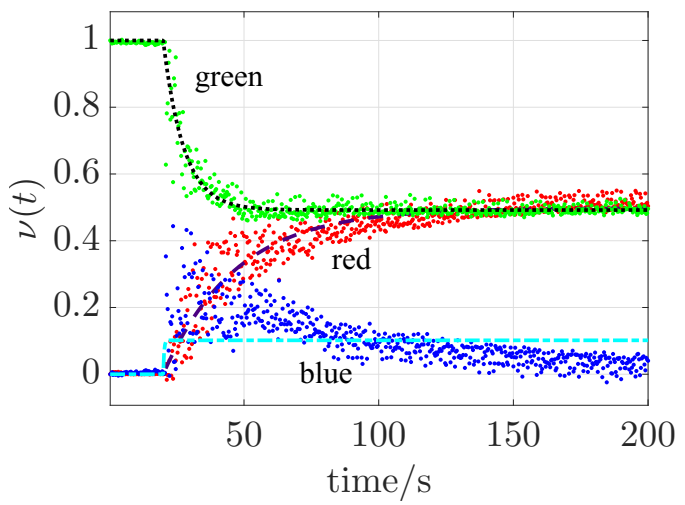

(c) $C_{2}$ uncalibrated.

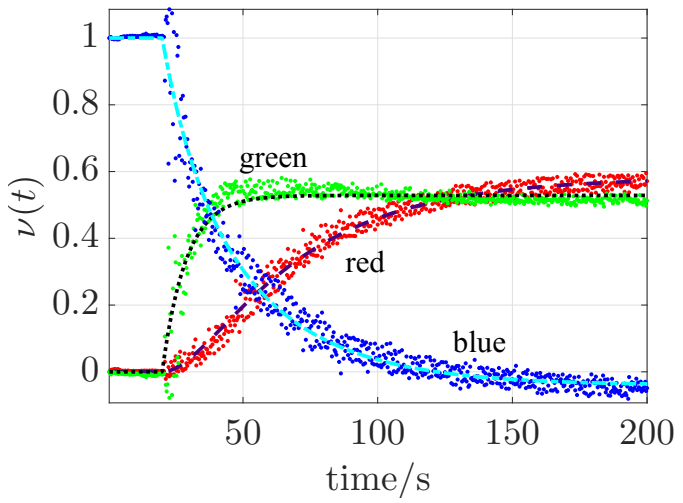

(e) $C_{3}$ uncalibrated.

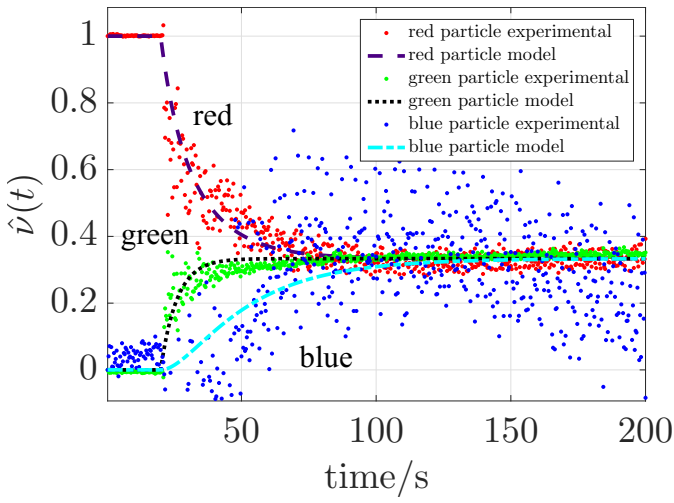

(b) $C_{1}$ calibrated.

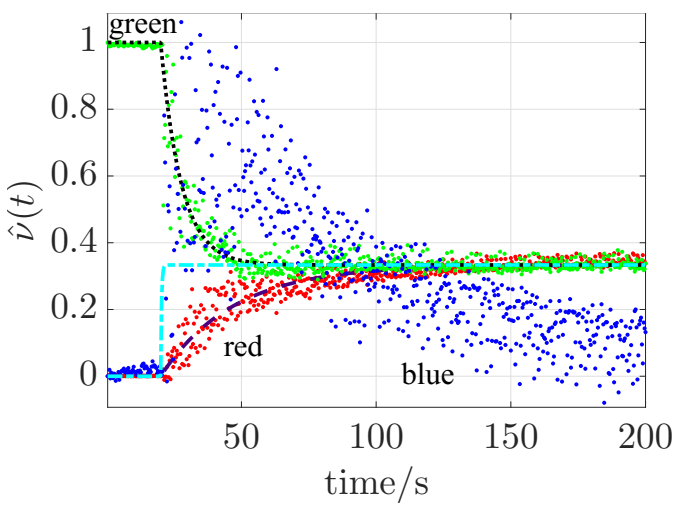

(d) $C_{2}$ calibrated.

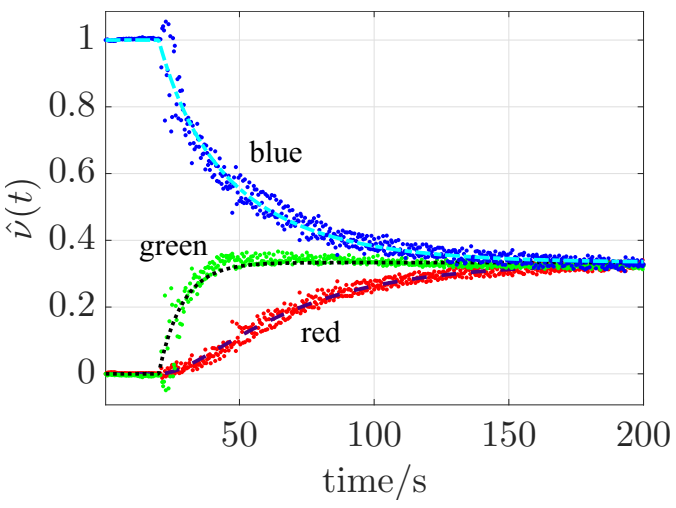

(f) $C_{3}$ calibrated.

Figure 10: Uncalibrated and calibrated measured volume fraction evolutions derived from the image analysis algorithm applied to the tertiary component wet granule system. The uncalibrated and calibrated model responses are included in all cases. 


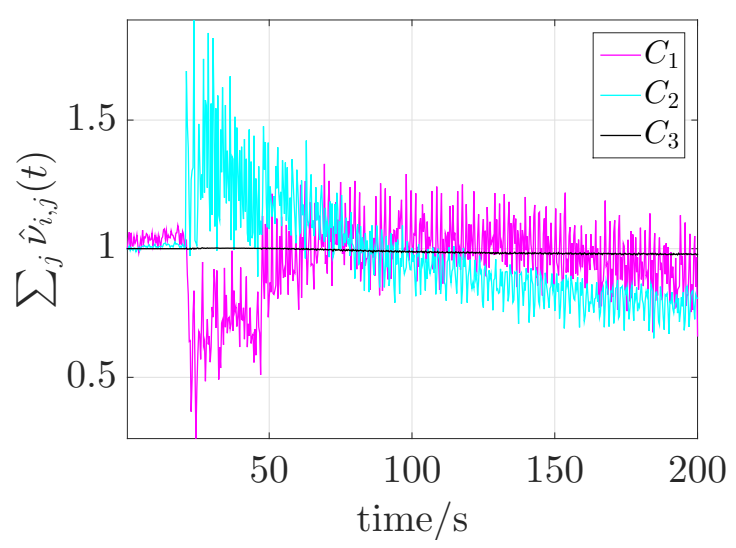

Figure 11: The calibrated volume fraction sum over each component within each compartment of the tertiary component system.

\subsubsection{Binary system}

The tertiary component colour mixing results indicate that a system with a higher degree of linear independence between pure component colour vectors would be advantageous in terms of reducing colour identification errors. To test this theory and further validate the residence time derived in the previous section a binary mixing experimental was carried out, again using the same granules with a three compartment system initially loaded with material as [red,blue,blue]. All other experimental conditions remained the same as the tertiary case. The resulting volume fraction evolutions from the image analysis routines are presented in Figure 13. It is noted that the volume fraction evolutions are subject to a much lower degree of noise than that observed in the tertiary case, with the possible exception of those in $C_{2}$ where this has lead to a poor model fit. The cause of the high degree of noise in $C_{2}$ is unclear but could be caused by large/temporary 'apparent' composition changes associated with radial inhomogeneities in the bed, breaking the relationship between surface area coverage and particle volume fraction. The noise may also be associated with the failure of the mixer removal algorithm to effectively detect/fully remove the mixer blades present in this area of the system.

It is noted from Figure 12 that the degree of calibration required in the binary mixing case in generally much lower than that of the tertiary system. Furthermore, only very limited breakage of continuity is observed across all compartments as illustrated by the significantly reduced $y$-axis scale of Figure 13(d) relative to the results of the tertiary system in Figure 11.

In terms of residence time values it is noted from Figure 12 that the $\tau$ values derived from the particle volume fraction evolution in $C_{3}$ are in very good agreement with the result obtain in the tertiary mixing case with $\tau \approx 41 \mathrm{~s}$. The $\tau$ values evaluated in $C_{1}$ are much lower than those observed in $C_{3}$. This is expected since the image analysis algorithm cannot differentiate between blue particles originating in the $C_{2}$ and blue particles originating in the $C_{3}$. It is noted that the initial increase of the blue volume fraction in Figure 13(c) and the corresponding decrease of the red particle volume fraction at $t \approx \theta$ is likely the 
result of surface imperfections in terms of initial material segregation, which would subsequently 'dilute' the pure component basis vectors. This dilution is apparent in $C_{2}$ where the system appears slightly impure at $t<\theta$.

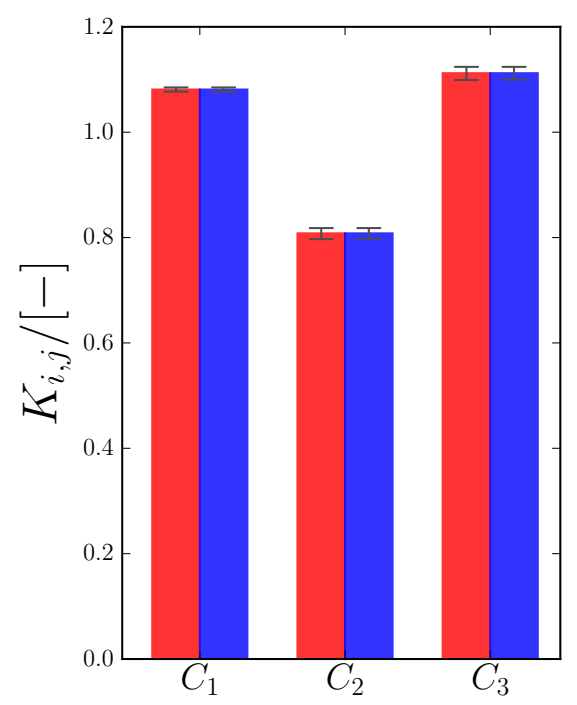

(a)

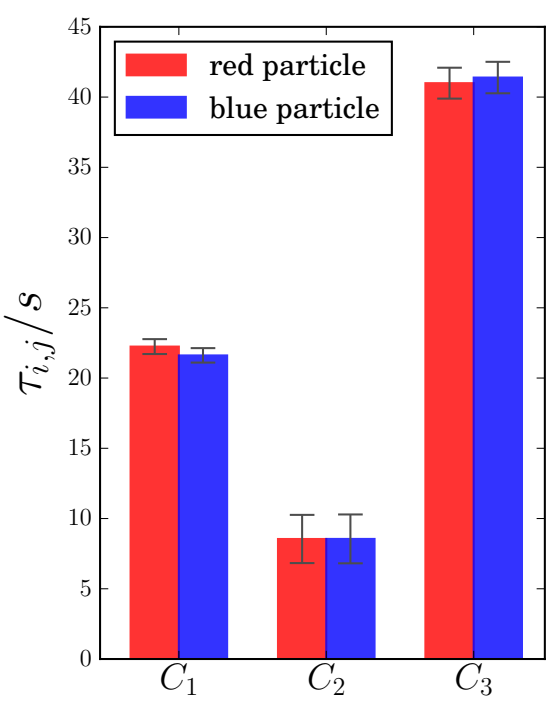

(b)

Figure 12: Fitted model parameters derived from the binary component mixing experiment with $95 \%$ confidence intervals. 


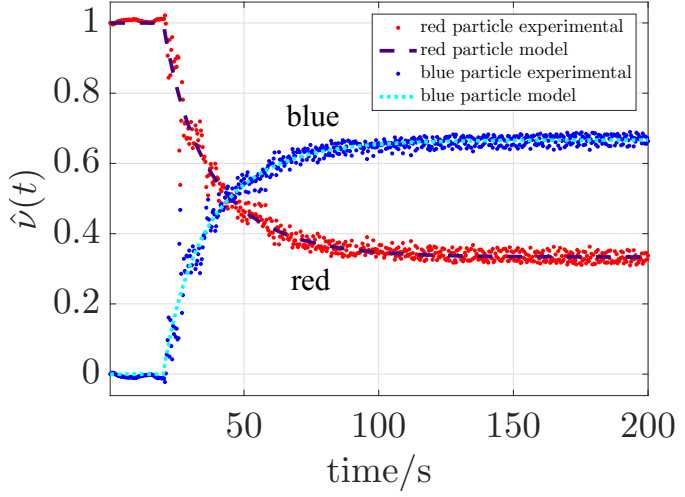

(a) $C_{1}$ calibrated.

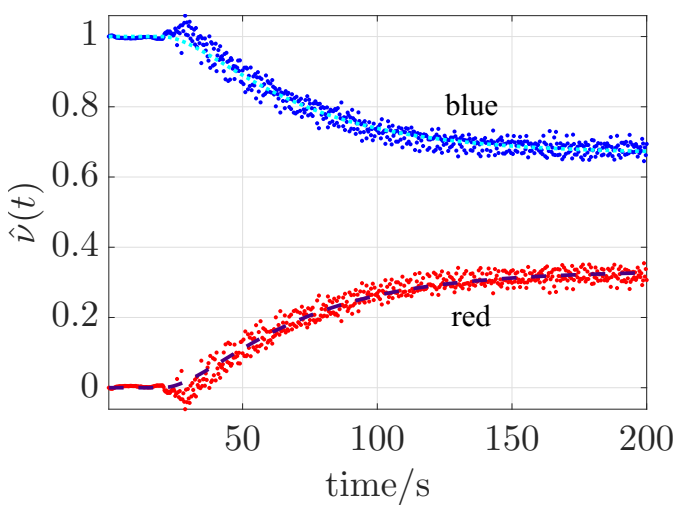

(c) $C_{3}$ calibrated.

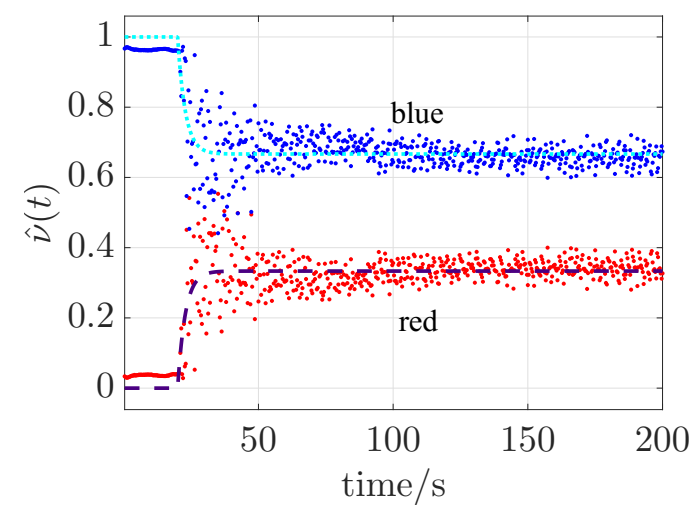

(b) $C_{2}$ calibrated.

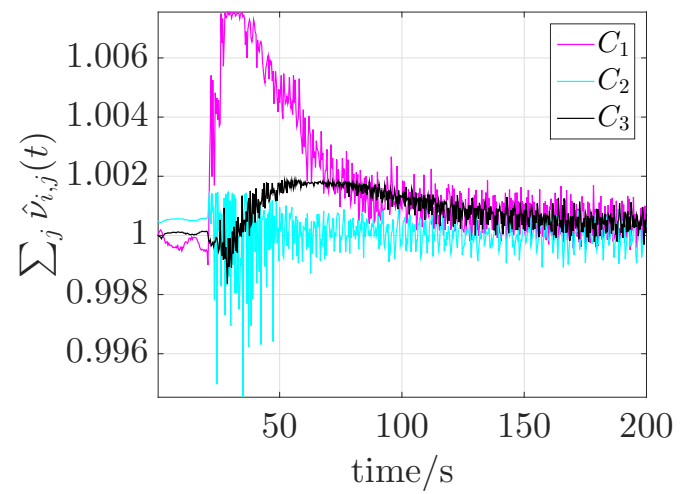

(d) $C_{3}$ calibrated.

Figure 13: Calibrated volume fraction evolutions derived from the image analysis algorithm applied to the binary component wet granule mixing system $(a)-(c)$. The calibrated volume fraction sum over each component within each compartment of the binary component system as a function of time $(d)$. 


\subsection{DEM results}

As mentioned in Section 2.3, two DEM simulations with different size distributions are carried out, one with uniform particles and the other with an experimental PSD. The computational cost of the DEM simulation increases as the range of simulated particle size increases even with the number of particles held constant. It took about two weeks to simulate the case with uniform size for 200 seconds, however the simulation with the experimental PSD requires 5 days for 10 seconds. Therefore, only 100 seconds worth of data are shown for the case with PSD.

Two types of analysis are presented for the DEM results. First, in order to ascertain the appropriateness of examining the particle bed from the surface as it is done in the image analysis experiments, the volume fractions derived from the particle bed surface in DEM is compared with the true volume fractions as calculated using Eq. (18). Only the simulation with uniform particles is used for this analysis.

In the second part of this section, the volume fractions from the DEM simulations (uniform particle and PSD) are directly compared with the image analysis experiments.

\subsubsection{Appropriateness of examining particle concentrations from the surface}

Snapshots from the DEM simulation with uniform particles at $t=0,100,200$ seconds are shown in Figure 14. From the images, it can be seen that the colours are fairly homogeneous in the radial direction.

As shown in Figure 14, the particle bed will be tilted at an angle in the direction of rotation during operation. In order to determine the angle of the surface accurately, axially averaged particle occupancies in $5 \mathrm{~mm} \times 5 \mathrm{~mm}$ pixels are plotted in Figure 15(a) for the case with uniform particles.

Particle occupancies are traditionally used in PEPT (Positron Emission Particle Tracking) studies to assess the overall flow behaviour and calculated as the fraction of time spent in a cell $[36,37,41,42]$. However, in this work, the occupancy, $O$, for a pixel $p$ is defined as

$$
O(p)=\frac{1}{T}\left[\sum_{t=1}^{T} \frac{N(t, p)}{N_{\text {total }}}\right]
$$

where $N(t, p)$ is the number of particles occupying pixel $p$ at time $t$ and $N_{\text {total }}$ is the total number of particles in the system. From the definition, it can be deduced that the occupancies are calculated as the number fraction of particles occupying each pixel and the values are averaged over 200 snapshots for the duration of $T=200$ seconds. Unlike PEPT, the time step in the DEM simulation is much larger, making the calculation of time fraction infeasible because particles may have hopped across multiple cells in a single time step. However, DEM is able to track all the particles in the system simultaneously, making the calculation of number fractions in the pixels feasible. This approach should yield the same information as the approach used in the PEPT studies because it shows where particles are located on average.

The colour concentrations at the surface are then calculated by considering particles located in the slice shown in Figure 15(a) and the particles outside this zone are ignored. 
The comparison between the volume fractions calculated from the surface and the bulk volume fractions for $C_{3}$ is presented in Figure 15(b) (similar trends can be observed for the other compartments). It is clear that the volume fractions derived from the surface are quite close to the bulk volume fractions. From here, it can be concluded that the mixing is fairly homogeneous in the radial direction and it is safe to assume that the colour concentrations observed from the bed surface are representative of the true concentrations in the image analysis experiments.

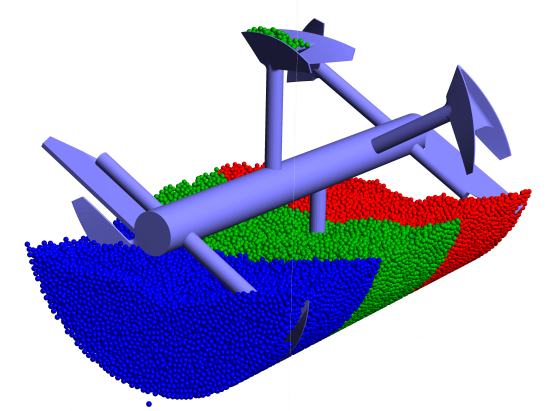

(a) $t=0 \mathrm{~s}$

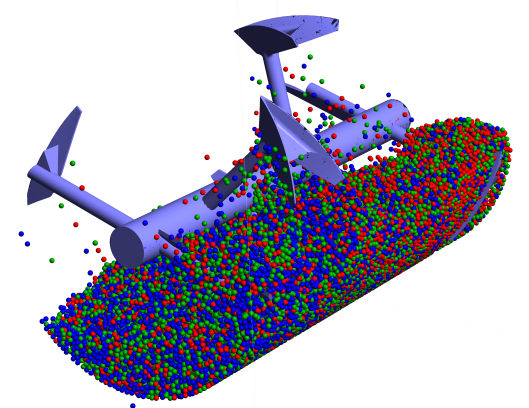

(b) $t=100 \mathrm{~s}$

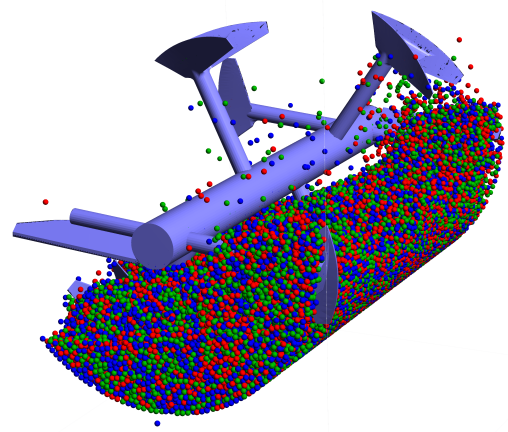

(c) $t=200 \mathrm{~s}$

Figure 14: Snapshots of the DEM simulation with coloured particles.

\subsubsection{Comparison with experimental data}

In this section, the volume fractions from the DEM simulations are directly compared against the experimentally measured values. Figure 16 compares the volume fractions calculated from the DEM simulation with uniform particles against the image analysis experiments. The results of the mixing model fitting procedure are presented in Figure 17. From the plots in both Figure 16 and 17, it can be observed that the DEM simulation with uniform particles takes longer to mix compared to the experiments as the volume fractions take longer to achieve the equivalent values from the experiments. It is suspected that the reason that the DEM simulation mixes slower than the experiments is due to the absence of large particles in the system. Another potential factor is fact that the water content on 
the surface of the experimental granules is not represented in the DEM simulations. This surface moisture has the potential to effect the flow behaviour of the particle bed.

Next, the effects of having PSD in the DEM simulation are shown in Figure 18 for $C_{3}$. From the plots, it can be observed that when the experimental PSD is used instead of uniform particles, the agreement between the experimental values and simulation results improves. The effect of a large particle(s) moving between compartments $C_{3}$ and $C_{2}$ can also be observed from the sharp change in green particle concentration in Figure 18(c). These results show that PSD plays an important role in determining the flow behaviour in such systems and it should not be ignored. 

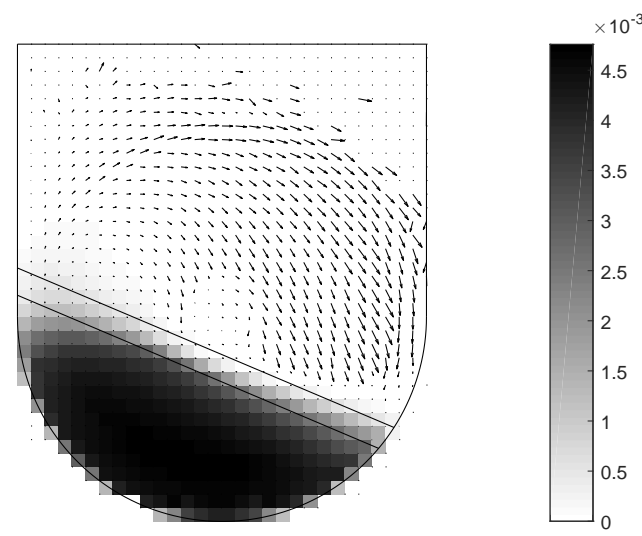

(a) Occupancy (uniform particles).

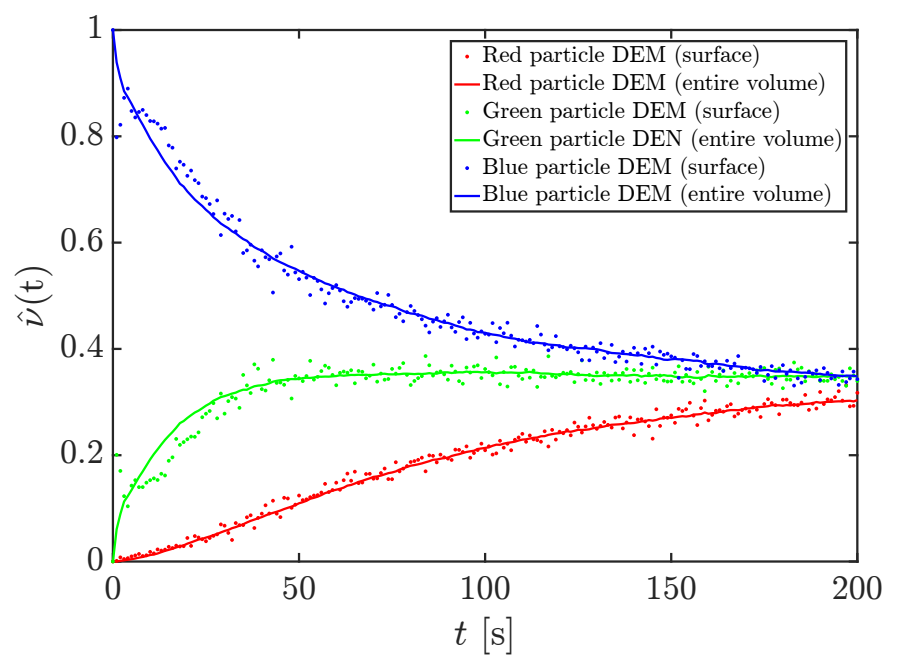

(b) Concentration evolution comparison (uniform particles).

Figure 15: (a) Plot showing pixels shaded according particle occupancies and velocity arrows proportional to mean velocities. The slice used to calculate the surface volume fractions is also shown. (b) A comparison of the concentration gradients derived from the surface/bulk material in $C_{3}$. 


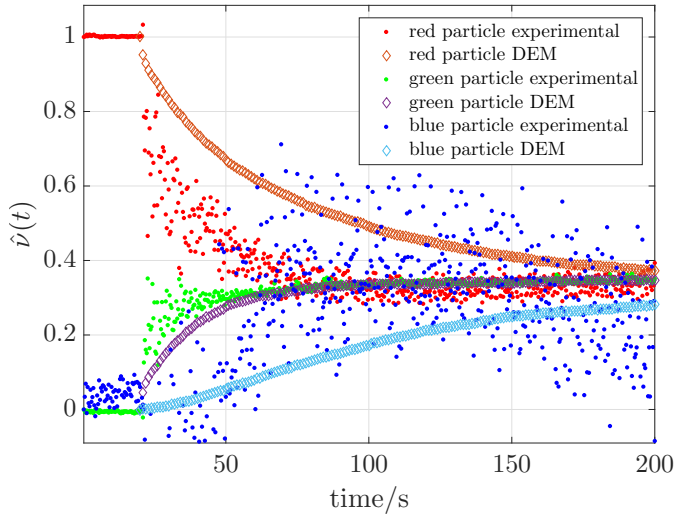

(a) $C_{1}$ DEM uniform particles (tertiary)

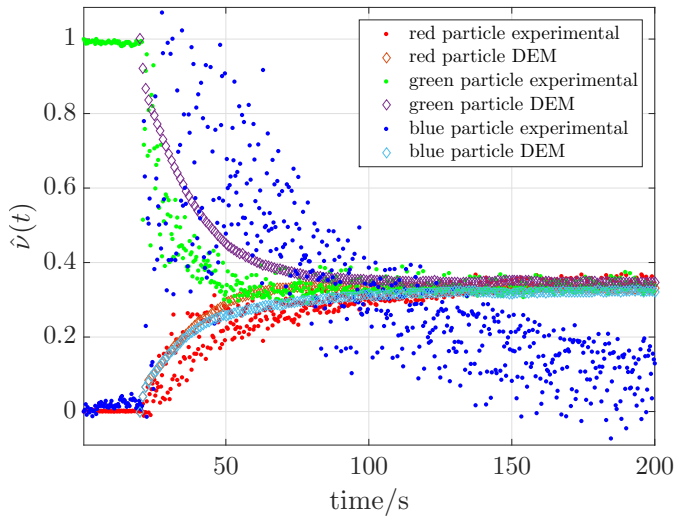

(c) $C_{2}$ DEM uniform particles (tertiary)

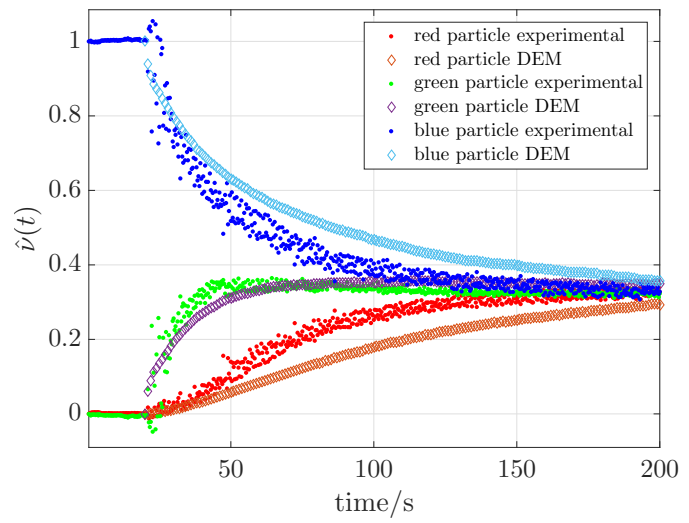

(e) $C_{3}$ DEM uniform particles (tertiary)

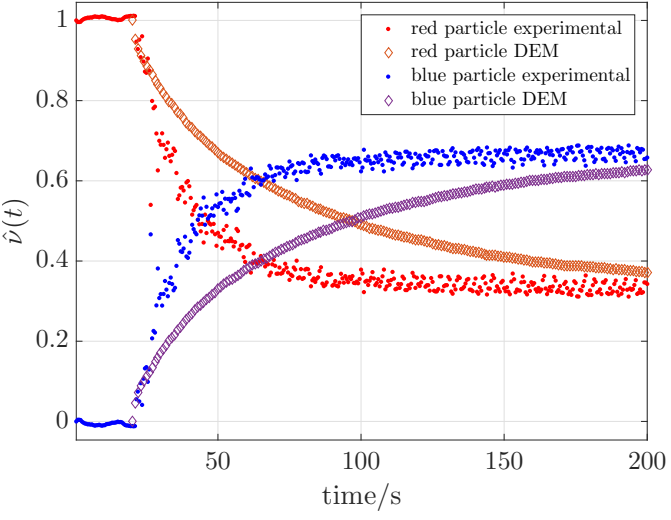

(b) $C_{1}$ DEM uniform particles (binary)

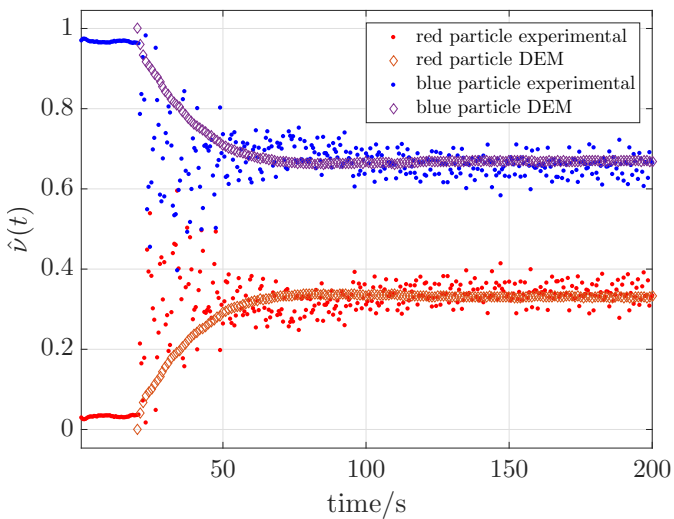

(d) $C_{2}$ DEM uniform particles (binary)

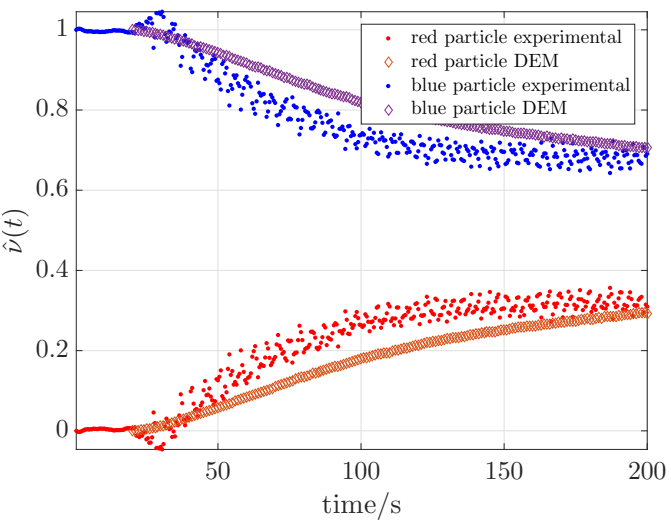

(f) $C_{3}$ DEM uniform particles (binary)

Figure 16: DEM vs. colourimetric experiment 


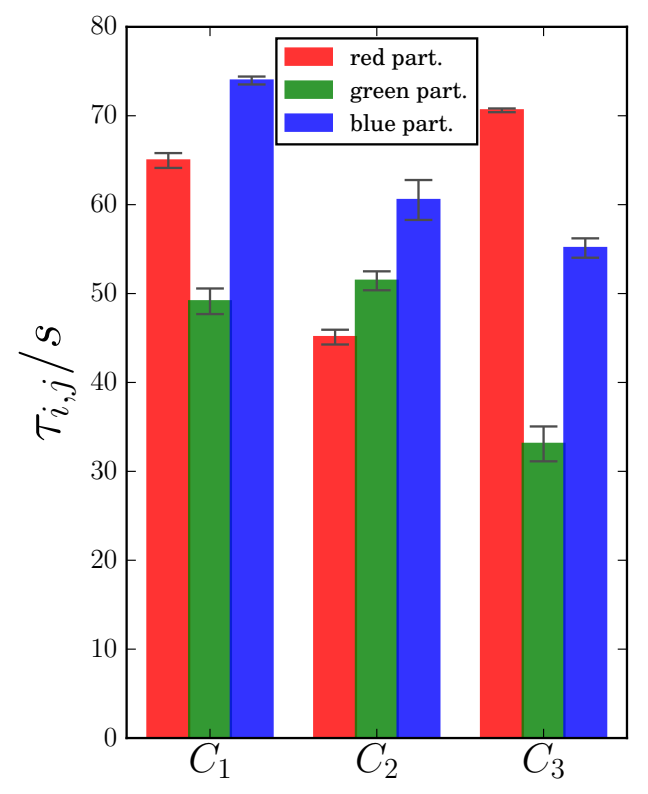

(a) as tertiary

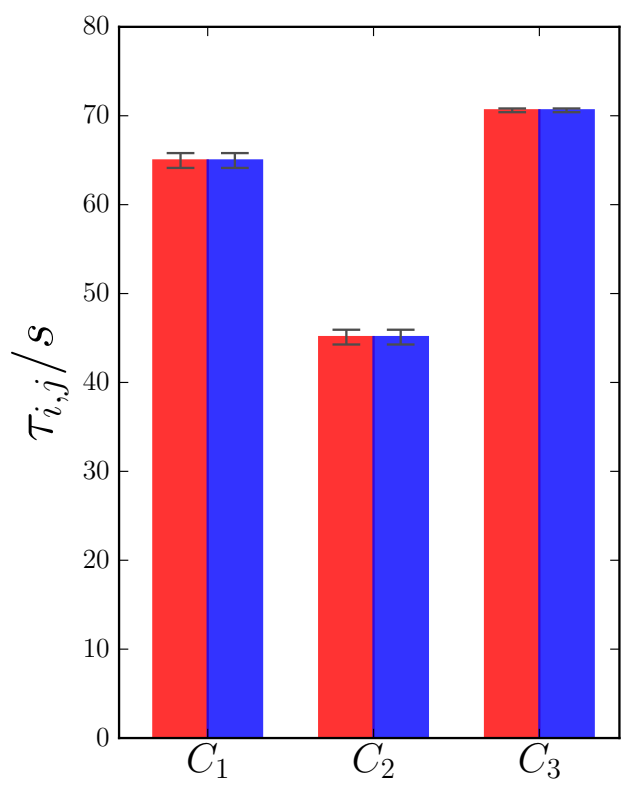

(b) as binary

Figure 17: Fitted mixing model parameters with 95\% confidence intervals for DEM simulations with uniform particle size. For all fits $K_{i, j}$ was set to unity and $\theta=20$ $s$. 


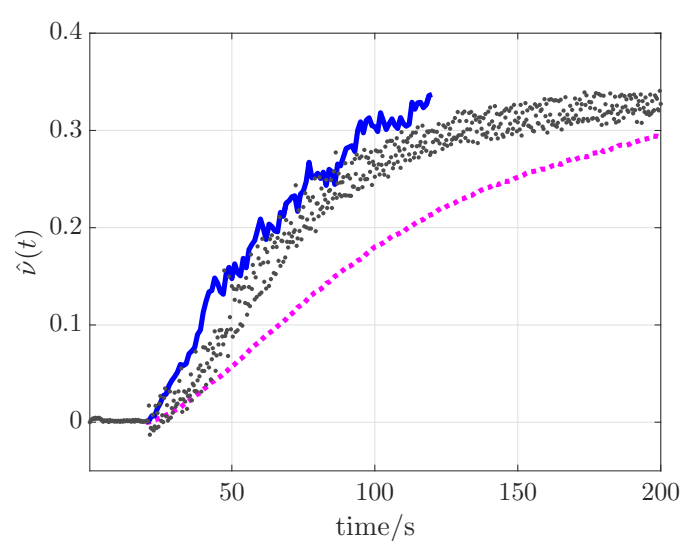

(a) red particles

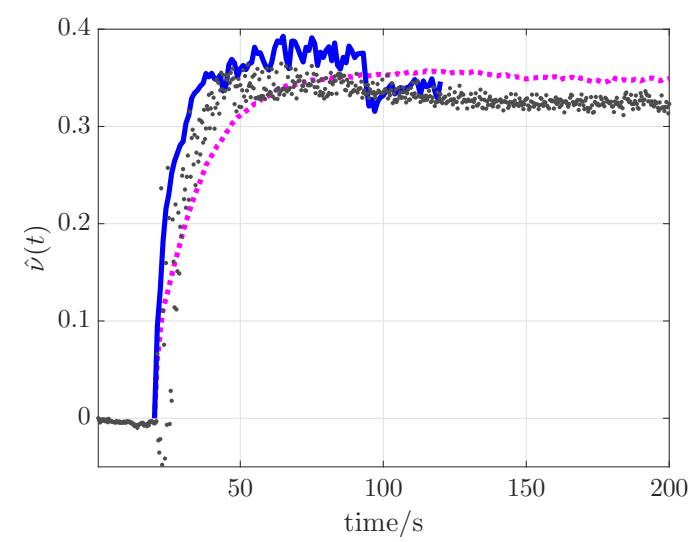

(b) green particles

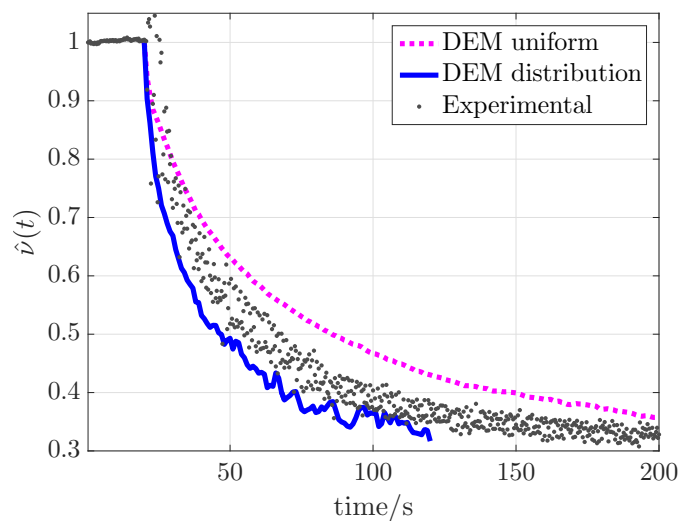

(c) blue particles

Figure 18: The effect of DEM particle size as seen from a tertiary particle colour evolutions in compartment 3. 


\section{Conclusions}

We have presented an optical experimental method for the estimation of residence times within regions of non-ideal batch granular systems. This technique involves the use of coloured agglomerates which induced spatial colour changes when mixed, which are then translated to changes in particle volume fractions and finally to compartment residence times. A proof of concept of the complete algorithm, from experiment to residence time extraction, has been carried out over tertiary and binary coloured granular systems. Though key residence times between the two wet systems were in agreement, we have found the proposed algorithms to be most stable in the case of the binary particle colour system. The mixing models developed for the extraction of residence times in this study agreed well with the observed experimental trends. Disparities in observed residence times between particle colour types in the granular system highlighted that the compartments within the mixer could not be strictly treated as perfectly mixed. Experimental observations also highlighted the potential for the underestimation of residence times due to layering on larger particles. We observe that particle colour identification errors are reduced through the use of more distinct particle colouring.

In addition to the image analysis experiments, we also carried out DEM simulations with two different particle distributions to validate the optical experimental method. One of the key findings from the results is that the system is very well-mixed in the radial direction and it is safe to assume that the colour concentrations observed from the bed surface are representative of the true concentrations. Besides that, the volume fractions calculated from the simulations are also directly compared with the experimental results and we showed that it is important to consider the real particle size distribution while simulating such systems.

\section{Acknowledgements}

The authors would like to thank AstraZeneca for funding this work. This project was partly funded by the National Research Foundation (NRF), Prime Minister's Office, Singapore under its Campus for Research Excellence and Technological Enterprise (CREATE) programme.

\section{Nomenclature}

\section{Roman symbols}

$b$ blue component of an rgb vector

$B$ blue component of an RGB vector [-]

$C_{i} \quad$ compartment $i \quad[-]$

$g$ green component of an rgb vector $\quad[-]$

$G$ green component of an RGB vector [-] 


$\begin{array}{lll}H & \text { Heaviside step function } & {[-]} \\ K_{i, j} & \text { fitted model gain particle } j \text { in compartment } i & {[-]} \\ \dot{M}_{k \rightarrow p} & \text { mass flow-rate from compartment } k \text { to } p & \mathrm{~kg} \mathrm{~s}^{-1} \\ n & \text { number of compartments } & {[-]} \\ N & \text { number of particles } & {[-]} \\ O & \text { occupancy } & {[-]} \\ Q_{k \rightarrow p} & \text { volumetric flow-rate from compartment } k \text { to } p & \mathrm{~m}^{3} \mathrm{~s}^{-1} \\ Q_{k \rightarrow p}^{[j]} & \text { volumetric flow-rate of particle type } j \text { from compartment } k \text { to } p & \mathrm{~kg} \mathrm{~s}^{-1} \\ r & \text { red component of an rgb vector } & {[-]} \\ R & \text { red component of an RGB vector } & {[-]} \\ \text { RGB } & \text { Three component digital RGB column vector } & {[-]} \\ t & \text { time } & \mathrm{S} \\ V_{i, T} & \text { total volume of particles in compartment } i & \mathrm{~m}^{3} \\ V_{\text {particle }} & \text { total volume of particles } & \mathrm{m}^{3}\end{array}$

\section{Greek symbols}

$\theta \quad$ model time-delay

$\lambda_{i} \quad$ normalised average RGB vector for compartment $i$

$v_{i, j} \quad$ uncalibrated particle volume fraction for particle type $j$ in compartment

$\boldsymbol{\nu}_{i} \quad$ vector containing uncalibrated particle volume fractions for all compo- $[-]$ nents in compartment $i$

$\hat{v}_{i, j} \quad$ calibrated particle volume fraction for particle type $j$ in compartment $i$

$\hat{\boldsymbol{\nu}}_{i} \quad$ vector containing calibrated particle volume fractions for all components in compartment $i$

$\tau_{i} \quad$ characteristic residence time of compartment $i$

$\phi_{i, j} \quad$ colour calibration constant for particle type $j$ in compartment $i$

$\boldsymbol{\Phi}_{i} \quad$ colour calibration array for compartment $i$

\section{Supplementary material}

A model for each particle volume fraction evolution in each compartment is derived by considering an idealised reactor system as shown in Figure 19. Here, a tertiary particle colour system is initially loaded as [red, green, blue] and each compartment is perfectly mixed. Such a construction assumes that the separation between compartment boundaries is sufficiently large and the time step between analysis frames is sufficiently small such that particles cannot 'skip' over compartments (e.g. from $C_{1}$ directly to $C_{3}$ ). The mass flow rate from compartment $k$ to $p$ can then be written as the sum of the time-dependent component mass flow-rates $R_{k \rightarrow p}^{[j]}(t)$ as:

$$
R_{k \rightarrow p}=R_{k \rightarrow p}^{[\mathrm{red}]}(t)+R_{k \rightarrow p}^{[\mathrm{green}]}(t)+R_{k \rightarrow p}^{[\mathrm{blue}]}(t)
$$

Since the mixer is being operated at a constant speed we expect the mass transfer rate (driven by the energy supplied by the motor) to be constant with respect to time (neglecting the time taken to establish any potential particle flow channels). Assuming constant 


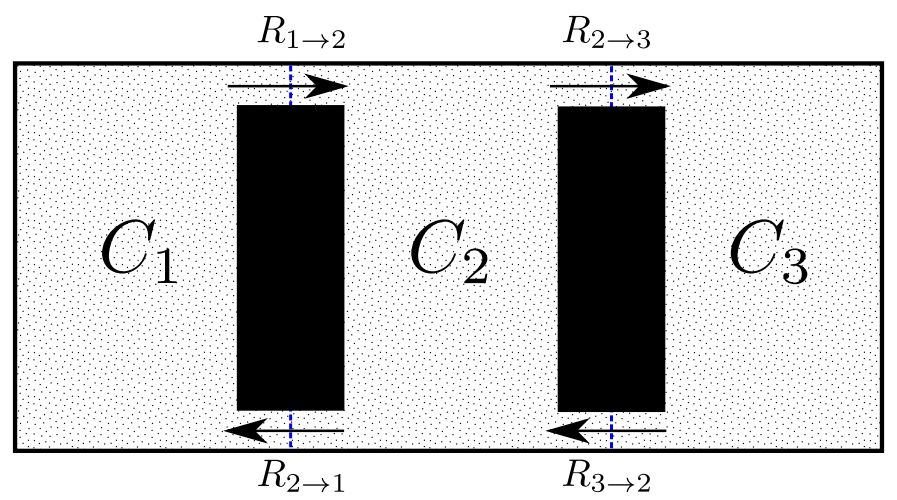

Figure 19: Idealised compartmental mixer in which particles travel at a constant rate through pre-defined channels. Each compartment acts as a well mixed CSTR.

mass hold-up within each compartment (no net movement between compartments was observed during the experiment) it follows that, for compartments connected to a single other compartment (compartment 1 and 3 in Figure 19),

$$
R_{k \rightarrow p}=R_{p \rightarrow k} .
$$

Furthermore, mixer symmetry dictates that:

$$
R_{1 \rightarrow 2}=R_{3 \rightarrow 2},
$$

and thus:

$$
R_{1 \rightarrow 2}=R_{2 \rightarrow 1}=R_{2 \rightarrow 3}=R_{3 \rightarrow 2} .
$$

If particles have a uniform density across the system then the corresponding volumetric flowrates $Q$ may be written as:

$$
Q_{1 \rightarrow 2}=Q_{2 \rightarrow 1}=Q_{2 \rightarrow 3}=Q_{3 \rightarrow 2} .
$$

By definition the average residence time $\tau$ of a compartment is given by:

$$
\tau_{i}=\frac{V_{i, T}}{Q_{i, o u t}},
$$

where $V_{i, T}$ and $Q_{i, o u t}$ are the total particle volume and volumetric flow-rate out of compartment $i$, respectively. The compartments are constructed such that $V_{1, T}=V_{2, T}=V_{3, T}$ and $Q_{1, \text { out }}=Q_{2, \text { out }} / 2=Q_{3, \text { out }}$, and so:

$$
\tau_{1}=2 \tau_{2}=\tau_{3}
$$

Again, assuming constant particle density, a dynamic component balance over the red particles in $C_{1}$ may be written as:

$$
\frac{\mathrm{d} V_{1, \text { red }}(t)}{\mathrm{d} t}=Q_{2 \rightarrow 1}^{[\mathrm{red}]}-Q_{1 \rightarrow 2}^{[\mathrm{red}]}
$$


If half of the red particles in $C_{2}$ are assumed to exit into $C_{1}$ and half through to $C_{3}$ (again this assumes that $C_{2}$ is well mixed) then it follows from (27) that:

$$
\begin{aligned}
V_{1, T} \frac{\mathrm{d} \hat{v}_{1, \text { red }}(t)}{\mathrm{d} t}=\frac{V_{2, \text { red }}}{2 \tau_{2}}-\frac{V_{1, \text { red }}}{\tau_{1}} \\
\rightarrow \frac{\mathrm{d} \hat{v}_{1, \text { red }}(t)}{\mathrm{d} t}=\frac{1}{\tau_{1}}\left(\hat{v}_{2, \text { red }}(t)-\hat{v}_{1, \text { red }}(t)\right) .
\end{aligned}
$$

A similar argument applied to $C_{2}$ and $C_{3}$ yields:

$$
\begin{aligned}
& \frac{\mathrm{d} \hat{v}_{2, \text { red }}(t)}{\mathrm{d} t}=\frac{1}{\tau_{1}}\left(\hat{v}_{1, \text { red }}(t)+\hat{v}_{3, \text { red }}(t)-2 \hat{v}_{2, \text { red }}(t)\right), \\
& \frac{\mathrm{d} \hat{v}_{3, \text { red }}(t)}{\mathrm{d} t}=\frac{1}{\tau_{1}}\left(\hat{v}_{2, \text { red }}(t)-\hat{v}_{3, \text { red }}(t)\right) .
\end{aligned}
$$

Equations (28), (29) and (30) form a system of ordinary differential equations with initial conditions $\hat{v}_{1, \text { red }}(0)=1$ and $\hat{v}_{2, \text { red }}(0)=\hat{v}_{3 \text {,red }}(0)=0$. The solutions to the system are then:

$$
\begin{aligned}
& \hat{v}_{1, \text { red }}(t)=\frac{1}{2} \exp \left(-\frac{t}{\tau_{1}}\right)+\frac{1}{6} \exp \left(-\frac{3 t}{\tau_{1}}\right)+\frac{1}{3} \\
& \hat{v}_{2, \text { red }}(t)=-\frac{1}{3} \exp \left(-\frac{3 t}{\tau_{1}}\right)+\frac{1}{3}, \\
& \hat{v}_{3, \text { red }}(t)=-\frac{1}{2} \exp \left(-\frac{t}{\tau_{1}}\right)+\frac{1}{6} \exp \left(-\frac{3 t}{\tau_{1}}\right)+\frac{1}{3} .
\end{aligned}
$$

Due to the symmetrical nature of both the mixture operation and loading it is known that in an ideal system:

$$
\begin{aligned}
& \hat{v}_{1, \text { blue }}(t)=\hat{v}_{3, \text { red }}(t), \\
& \hat{v}_{2, \text { blue }}(t)=\hat{v}_{2, \text { red }}(t), \\
& \hat{v}_{3, \text { blue }}(t)=\hat{v}_{1, \text { red }}(t) .
\end{aligned}
$$

Furthermore, from the definition of volume fraction we have:

$$
\hat{v}_{i, \text { green }}(t)=1-\hat{v}_{i, \text { red }}(t)-\hat{v}_{i, \text { blue }}(t)
$$

for each compartment $i$. Again acknowledging the mixer symmetry, it follows that:

$$
\begin{aligned}
& \hat{v}_{1, \text { green }}(t)=\hat{v}_{3, \text { green }}(t)=\hat{v}_{2, \text { red }}(t)=\hat{v}_{2, \text { blue }}(t), \\
& \hat{v}_{2, \text { green }}(t)=\frac{2}{3} \exp \left(-\frac{3 t}{\tau_{1}}\right)+\frac{1}{3}
\end{aligned}
$$

Introducing a predefined time-delay $\theta$ to model the constant composition of the system prior to switching on the mixer and converting from volume fraction to volume fraction 
change form, the four unique model equations become:

$$
\begin{aligned}
\Delta \hat{v}_{1, \text { red }}(t)_{\text {model }} & =\left[\frac{1}{2} \exp \left(-\frac{(t-\theta)}{\tau_{1}}\right)+\frac{1}{6} \exp \left(-\frac{3(t-\theta)}{\tau_{1}}\right)-\frac{2}{3}\right] H(t-\theta), \\
\Delta \hat{v}_{2, \text { red }}(t)_{\text {model }} & =\left[-\frac{1}{3} \exp \left(-\frac{3(t-\theta)}{\tau_{1}}\right)+\frac{1}{3}\right] H(t-\theta), \\
\Delta \hat{v}_{3, \text { red }}(t)_{\text {model }} & =\left[-\frac{1}{2} \exp \left(-\frac{(t-\theta)}{\tau_{1}}\right)+\frac{1}{6} \exp \left(-\frac{3(t-\theta)}{\tau_{1}}\right)+\frac{1}{3}\right] H(t-\theta), \\
\Delta \hat{v}_{2, \text { green }}(t)_{\text {model }} & =\left[\frac{2}{3} \exp \left(-\frac{3(t-\theta)}{\tau_{1}}\right)-\frac{2}{3}\right] H(t-\theta),
\end{aligned}
$$

where $H$ is the Heaviside step function. We now have a complete set of equations to describe the calibrated volume fraction evolution of each particle colour in each compartment of the system. These are graphically illustrated for selected parameter values in Figure 20. These models are easily combined to model binary particle colour systems and a variety of loading patterns. Furthermore, such a modelling procedure could also be carried out on alternative/more complex granulation/mixing equipment.

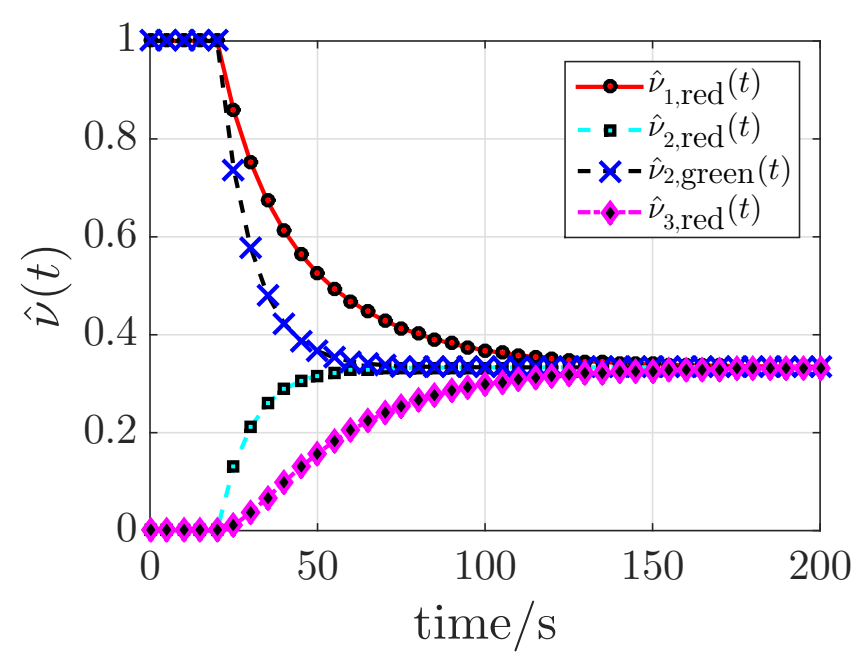

Figure 20: Theoretical model responses from Eqns. (36)-(39) for a tertiary system initial loaded from left to right as [red,green,blue] with $\tau_{1}=30 \mathrm{~s}$ and $\theta=20 \mathrm{~s}$. 


\section{References}

[1] A. A. Aissa, C. Duchesne, and D. Rodrigue. Polymer powders mixing part II: Multicomponent mixing dynamics using RGB color analysis. Chem. Eng. Sci., 65(12): 3729-3738, 2010.

[2] M. Alian, F. Ein-Mozaffari, and S. R. Upreti. Analysis of the mixing of solid particles in a plowshare mixer via discrete element method (dem). Powder Technol., 274: $77-87,2015$.

[3] P. Bannister and N. Harnby. A colorimetric technique for assessing the mixture quality of fine particle mixtures. Powder Technol., 36(2):275-279, 1983.

[4] D. Barrasso, S. Walia, and R. Ramachandran. Multi-component population balance modeling of continuous granulation processes: A parametric study and comparison with experimental trends. Powder Technol., 241:85 - 97, 2013.

[5] D. Barrasso, A. E. Hagrasy, J. D. Litster, and R. Ramachandran. Multi-dimensional population balance model development and validation for a twin screw granulation process. Powder Technol., 270, Part B:612 - 621, 2015.

[6] D. Barrasso, A. Tamrakar, and R. Ramachandran. Model order reduction of a multiscale PBM-DEM description of a wet granulation process via ANN. Procedia Eng., 102:1295 - 1304, 2015.

[7] G. Basinskas and M. Sakai. Numerical study of the mixing efficiency of a ribbon mixer using the discrete element method. Powder Technol., 287:380-394, 2016.

[8] R. Boerefijn, Z. Ning, and M. Ghadiri. Disintegration of weak lactose agglomerates for inhalation applications. Int. J. Pharm., 172(1-2):199 - 209, 1998.

[9] J. Bouffard, F. Bertrand, and J. Chaouki. A multiscale model for the simulation of granulation in rotor-based equipment. Chem. Eng. Sci., 81:106 - 117, 2012.

[10] A. Braumann and M. Kraft. Incorporating experimental uncertainties into multivariate granulation modelling. Chem. Eng. Sci., 65(3):1088 - 1100, 2010.

[11] A. Braumann, M. J. Goodson, M. Kraft, and P. R. Mort. Modelling and validation of granulation with heterogeneous binder dispersion and chemical reaction. Chem. Eng. Sci., 62(17):4717 - 4728, 2007.

[12] A. Braumann, M. Kraft, and P. R. Mort. Parameter estimation in a multidimensional granulation model. Powder Technol., 197(3):196 - 210, 2010.

[13] A. Braumann, M. Kraft, and W. Wagner. Numerical study of a stochastic particle algorithm solving a multidimensional population balance model for high shear granulation. J. Comput. Phys., 229(20):7672 - 7691, 2010. 
[14] A. Braumann, P. L. W. Man, and M. Kraft. Statistical approximation of the inverse problem in multivariate population balance modeling. J. Ind. Eng. Chem., 49(1): 428-438, 2010.

[15] A. Braumann, P. L. W. Man, and M. Kraft. The inverse problem in granulation modeling-two different statistical approaches. AIChE Journal, 57(11):3105-3121, 2011.

[16] J. Bridgwater. Mixing of powders and granular materials by mechanical means-a perspective. Particuology, 10(4):397-427, 2012.

[17] J. Bridgwater, C. J. Broadbent, and D. J. Parker. Study of the influence of blade speed on the perfromance of a powder mixer using positron emission particle tracking. Trans. IChemE, 71:675-681, 1993.

[18] C. Broadbent, J. Bridgwater, D. Parker, S. Keningley, and P. Knight. A phenomenological study of a batch mixer using a positron camera. Powder Technol., 76(3):317 $-329,1993$.

[19] C. Broadbent, J. Bridgwater, and D. Parker. The effect of fill level on powder mixer performance using a positron camera. Chem. Eng. J. Bioch. Eng., 56(3):119 - 125, 1995.

[20] P. W. Cleary. Charge behaviour and power consumption in ball mills: sensitivity to mill operating conditions, liner geometry and charge composition. Int. J. Miner. Process., 63(2):79-114, 2001.

[21] P. W. Cleary. Industrial particle flow modelling using discrete element method. Eng. Computation, 26(6):698-743, 2009.

[22] P. W. Cleary. Particulate mixing in a plough share mixer using $\{$ DEM $\}$ with realistic shaped particles. Powder Technol., 248:103 - 120, 2013. Discrete Element Modelling.

[23] P. W. Cleary and M. D. Sinnott. Assessing mixing characteristics of particle-mixing and granulation devices. Particuology, 6(6):419 - 444, 2008.

[24] P. W. Cleary, G. Metcalfe, and K. Liffman. How well do discrete element granular flow models capture the essentials of mixing processes? Appl. Math. Model., 22 (12):995 - 1008, 1998.

[25] B. Crüger, V. Salikov, S. Heinrich, S. Antonyuk, V. Sutkar, N. Deen, and J. Kuipers. Coefficient of restitution for particles impacting on wet surfaces: An improved experimental approach. Particuology, 25:1 - 9, 2016.

[26] M. Dosta, S. Antonyuk, and S. Heinrich. Multiscale simulation of the fluidized bed granulation process. Chem. Eng. Technol., 35(8):1373-1380, 2012.

[27] A. Ford and A. Roberts. Colour space conversions, 1998. URL: http://www. poynton. com/PDFs/coloureq.pdf [Last accessed: 12 April 2016], 2011. 
[28] S. Forrest, J. Bridgwater, P. R. Mort, J. Litster, and D. J. Parker. Flow patterns in granulating systems. Powder Technol., 130(1âĂŞ3):91 - 96, 2003.

[29] B. Freireich, J. Li, J. Litster, and C. Wassgren. Incorporating particle flow information from discrete element simulations in population balance models of mixercoaters. Chem. Eng. Sci., 66(16):3592 - 3604, 2011.

[30] R. Gosselin, C. Duchesne, and D. Rodrigue. On the characterization of polymer powders mixing dynamics by texture analysis. Powder Technol., 183(2):177 - 188, 2008.

[31] N. Gossett and B. Chen. Paint inspired color mixing and compositing for visualization. In INFOVIS. IEEE Symposium, pages 113-118, 2004.

[32] C. S. Haase and G. W. Meyer. Modeling pigmented materials for realistic image synthesis. ACM T. Graphic. (TOG), 11(4):305-335, 1992.

[33] M. J. Hounslow, J. M. K. Pearson, and T. Instone. Tracer studies of high-shear granulation: II. population balance modeling. AIChE Journal, 47(9):1984-1999, 2001.

[34] R. Irizarry. Fast compartmental monte carlo simulation of population balance models: Application to nanoparticle formation in nonhomogeneous conditions. Ind. Eng. Chem. Res., 51(47):15484-15496, 2012.

[35] S. M. Iveson, J. D. Litster, K. Hapgood, and B. J. Ennis. Nucleation, growth and breakage phenomena in agitated wet granulation processes: a review. Powder Technol., 117(1-2):3 - 39, 2001.

[36] J. Jones and J. Bridgwater. A case study of particle mixing in a ploughshare mixer using positron emission particle tracking. Int. J. Miner. Process., 53(1):29 - 38, 1998.

[37] J. Jones, D. Parker, and J. Bridgwater. Axial mixing in a ploughshare mixer. Powder Technol., 178(2):73 - 86, 2007.

[38] C. A. Kastner, G. P. Brownbridge, S. Mosbach, and M. Kraft. Impact of powder characteristics on a particle granulation model. Chem. Eng. Sci., 97:282 - 295, 2013.

[39] A. Kumar, K. V. Gernaey, T. D. Beer, and I. Nopens. Model-based analysis of high shear wet granulation from batch to continuous processes in pharmaceutical production - A critical review. Eur. J. Pharm. Biopharm., 85(3, Part B):814 - 832, 2013.

[40] A. Kumar, J. Vercruysse, V. Vanhoorne, M. Toiviainen, P.-E. Panouillot, M. Juuti, C. Vervaet, J. P. Remon, K. V. Gernaey, T. D. Beer, and I. Nopens. Conceptual framework for model-based analysis of residence time distribution in twin-screw granulation. Eur. J. Pharm. Sci., 71:25 - 34, 2015. 
[41] B. Laurent. Scaling factors in granular flow - analysis of experimental and simulations results. Chem. Eng. Sci., 61(13):4138 - 4146, 2006.

[42] B. Laurent and P. Cleary. Comparative study by pept and dem for flow and mixing in a ploughshare mixer. Powder Technol., 228:171 - 186, 2012.

[43] K. F. Lee, S. Mosbach, M. Kraft, and W. Wagner. A multi-compartment population balance model for high shear granulation. Comput. Chem. Eng., 75:1 - 13, 2015.

[44] J. Li, B. Freireich, C. Wassgren, and J. D. Litster. A general compartment-based population balance model for particle coating and layered granulation. AIChE Journal, 58(5):1397-1408, 2012.

[45] S. Maronga and P. Wnukowski. Modelling of the three-domain fluidized-bed particulate coating process. Chem. Eng. Sci., 52(17):2915-2925, 1997.

[46] MATLAB. version 8.4.0.150421 (R2014b). The MathWorks Inc., Natick, Massachusetts, 2014.

[47] W. J. Menz, J. Akroyd, and M. Kraft. Stochastic solution of population balance equations for reactor networks. J. Comput. Phys., 256:615-629, 2014.

[48] M. K. Paavolaa, A. S. El-Hagrasy, J. D. Litster, and K. J. Leiviskäa. 3D population balance model for continuous twin screw granulator. Chem. Eng. Trans., 32, 2013.

[49] J. M. K. Pearson, M. J. Hounslow, and T. Instone. Tracer studies of high-shear granulation: I. experimental results. AIChE Journal, 47(9):1978-1983, 2001.

[50] M. Poux, P. Fayolle, J. Bertrand, D. Bridoux, and J. Bousquet. Powder mixing: Some practical rules applied to agitated systems. Powder Technol., 68(3):213 - 234, 1991.

[51] D. Ramkrishna. Population Balances: Theory and Applications to Particulate Systems in Engineering. Elsevier Science, 2000.

[52] A. Realpe and C. Velázquez. Image processing and analysis for determination of concentrations of powder mixtures. Powder Technol., 134(3):193-200, 2003.

[53] B. Remy, J. G. Khinast, and B. J. Glasser. Discrete element simulation of free flowing grains in a four-bladed mixer. AIChE Journal, 55(8):2035-2048, 2009.

[54] J.-G. Rosenboom, S. Antonyuk, S. Heinrich, and M. Kraft. Characterisation of lactose powder and granules for multivariate wet granulation modelling. Chem. Eng. Sci., 123:395 - 405, 2015.

[55] C. Sanders, A. Willemse, A. Salman, and M. Hounslow. Development of a predictive high-shear granulation model. Powder Technol., 138(1):18 - 24, 2003. World Congress of Particle Technology.

[56] C. F. Sanders, M. J. Hounslow, and F. J. D. III. Identification of models for control of wet granulation. Powder Technol., 188(3):255 - 263, 2009. 
[57] A. Santomaso, M. Olivi, and P. Canu. Mechanisms of mixing of granular materials in drum mixers under rolling regime. Chem. Eng. Sci., 59(16):3269-3280, 2004.

[58] M. Sen and R. Ramachandran. A multi-dimensional population balance model approach to continuous powder mixing processes. Adv. Powder Technol., 24(1):51-59, 2013.

[59] O. S. Sudah, D. Coffin-Beach, and F. Muzzio. Quantitative characterization of mixing of free-flowing granular material in tote (bin)-blenders. Powder Technol., 126 (2):191 - 200, 2002.

[60] M. Tkalcic, J. F. Tasic, et al. Colour spaces: perceptual, historical and applicational background. In Eurocon, 2003.

[61] W. Tu, A. Ingram, and J. Seville. Regime map development for continuous twin screw granulation. Chem. Eng. Sci., 87:315 - 326, 2013.

[62] C. Wightman, F. J. Muzzio, and J. Wilder. A quantitative image analysis method for characterizing mixtures of granular materials. Powder Technol., 89(2):165-176, 1996.

[63] G. R. Ziegler and C. A. Aguilar. Residence time distribution in a co-rotating, twinscrew continuous mixer by the step change method. J. Food Eng., 59(2-3):161 167, 2003. 